\title{
Research Paper \\ An Evaluation of the Contributions of the Triple Vulnerability Model to the Prediction of Emotional Disorders
}

\author{
Tohid Ranjbari ${ }^{1}$, Javad Karimi² ${ }^{2}$ Abolfazl Mohammadi³ ${ }^{3}$ Mohammad Reza Norouzi ${ }^{4}$
}

1. MSc. Student, Department of Psychology, Faculty of Psychology and Education, University of Tehran, Tehran, Iran.

2. PhD in Family Counseling, Assistant Professor, Department of Psychology, Faculty of Literature and Humanities, Malayer University, Malayer, Iran.

3. PhD in Clinical Psychology, Assistant Professor, Department of Psychiatry, Roozbeh Hospital, Tehran University of Medical Sciences, Tehran, Iran.

4. MSc. Student, Department of Psychology, Faculty of Education and Psychology, University of Tabriz, Tabriz, Iran.

Received: 21 Sep. 2016

Accepted: 24 Apr. 2017

Key words:

Triple vulnerability model, Emotional disorders, Normal people
Citation: Ranjbari T, Karimi J, Mohammadi A, Norouzi MR. [An Evaluation of the Contributions of the Triple Vulnerability Model to the Prediction of Emotional Disorders (Persian)]. Iranian Journal of Psychiatry and Clinical Psychology. 2018; 23(4):408423. https://doi.org/10.29252/nirp.ijpcp.23.4.408

\begin{abstract}
A B S T R A C T
Objectives The triple vulnerability model includes three types of vulnerability in terms of emotional disorders: general biological vulnerability, general psychological vulnerability, and disorder-specific psychological vulnerability. Since previous studies have mostly addressed only one of these components, this study was conducted to examine all the three components of the triple vulnerability model at the same time in patients suffering from emotional disorders and normal people.

Methods Using a causal-comparative design, 20 patients with obsessive-compulsive disorder, 20 patients with major depressive disorder, and 20 patients with generalized anxiety disorder were compared with 20 normal people (control group). The data were collected from March to June 2015. The patients and control group were selected through convenience sampling from the people attending private psychotherapy clinics in the city of Urmia and the normal people living in Urmia, respectively. The patients and control groups were matched with each other with regard to some demographic variables. A multivariate analysis of variance (MANOVA) was conducted to determine the differences between the patients and the control group.

Results Patients suffering from generalized anxiety disorder, obsessive-compulsive disorder, and major depressive disorder were more vulnerable to general biological vulnerability and general psychological vulnerability $(P=0.001)$. With regard to disorder-specific psychological vulnerabilities, a significant difference was found between the patients with generalized anxiety and the control group where patients being more intolerant of uncertainty $(\mathrm{P}=0.001)$. We also found that obsessive-compulsive patients had more thought-action fusion and major depressive patients had more dysfunctional attitudes compared with the control group.

Conclusion Because of the significant difference between patients with emotional disorders and control group in terms of the dimensions of the triple vulnerability model, it can be argued that this model could be envisaged as comprehensive and suitable etiological and diagnostic criteria for these patients.
\end{abstract}

\section{Extended Abstract}

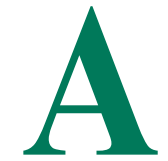

\section{Introduction}

nxiety disorders appear to be among the most common emotional disorders as epidemiological studies indicate that these disorders are the most prevalent mental disorders with an incidence rate of $16-29 \%$. Among these, Generalized Anxiety Disorder (GAD) is a common psychiatric disorder with an annual incidence rate of $3-8 \%$. The incidence rate of Obsessive Compulsive Disorder (OCD) reported to be $2-3 \%$ over a lifetime. Affecting $4.16 \%$ of the world population, Major Depressive Dis-

\section{* Corresponding Author:}

Javad Karimi, PhD

Address: Department of Psychology, Faculty of Literature and Humanities, Malayer University, Malayer, Iran.

Tel: +98 (913) 2756241

E-mail: dr.karimi10@yahoo.com 
order (MDD) is another prevalent psychiatric disorder referred to as the "common cold" of mental disorders. One of the most comprehensive models about etiology of these disorders is the triple vulnerability model. According to this model, etiology of emotional disorders is associated with three types of vulnerabilities: general biological vulnerability, general psychological vulnerability (perceived control over stressful events and emotional states), and disorder-specific psychological vulnerability (caused by individual learning experiences).

Despite the prominence of this model as a framework for understating the etiological features of emotional disorders, there is a dearth of research on a comprehensive evaluation of the dimensions of this model in Iran. Therefore, this study aimed to examine all three components of the triple vulnerability model in patients suffering from emotional disorders as well as normal people to shed more light on the role of each of these vulnerabilities in the etiology and explanation of emotional disorders. Additionally, such an evaluation is expected to provide researchers with a comprehensive examination of this model.

\section{Methods}

Employing a causal-comparative design, the present study was conducted from March 2014 to June 2015 . The statistical population of the study included all patients suffering from GAD, OCD, MDD, and the normal people living in Urmia. The participants $(\mathrm{N}=80)$ included 20 patients with GAD, 20 patients with OCD, 20 patients with MDD, and 20 normal people all of whom were recruited through convenience sampling. The patients were selected based on inclusion and exclusion criteria from people attending a private psychotherapy clinic in the city of Urmia. The normal people were also recruited from the public places of Urmia based on their volunteer participation. The patients and normal people were matched with each other to control for variables such as gender, marital status, and education. After receiving the initial diagnosis from a psychiatrist, the patients were given a structured clinical interview. In case their diagnosis was later confirmed, and they met the inclusion criteria, the patients were asked to sign an informed consent form and fill out the questionnaires individually. To gather the data, the following scales were used: NEO-FFI-R, Anxiety Control QuestionnaireRevised, Thought Fusion Instrument, Dysfunctional Beliefs Scale, and Intolerance of Uncertainty Scale. To analyze the data, a multivariate analysis of variance (MANOVA) was conducted using IBM SPSS 21.

\section{Results}

The results of the present study showed that there was a significant difference $(\mathrm{P}<0.001)$ among the four groups (patients with GAD, patients with OCD, patients with MDD, and control group) regarding the following variables: neuroticism, extraversion, anxiety control, thought-action fusion, dysfunctional attitudes, and the intolerance of uncertainty. The mean score of neuroticism in patients suffering from GAD, OCD, and MDD was higher than that in control group. However, the mean score of extraversion in patients with these disorders was lower than that in control group. Therefore, individuals suffering from emotional disorders were biologically more vulnerable than normal people. Patients with GAD, OCD, and MDD were also significantly different $(\mathrm{P}<0.001)$ from the control group in terms of anxiety control where the mean score of anxiety control in the patients suffering from GAD, OCD, and MDD being lower than that in control group. This variable is the main constituent of general psychological vulnerability. According to Barlow, individuals suffering from this vulnerability have a low perceived control over life stress and emotional states.

Additionally, the mean score of intolerance of uncertainty was highest in patients with GAD and MDD indicating a significant difference between these two groups and other groups. The other two variables, thought-action fusion and dysfunctional attitudes, demonstrated a high mean score in patients suffering from OCD and MDD, which were significantly different from other groups.

\section{Discussion}

The present study was undertaken to scrutinize the role played by the components of the triple vulnerability model in causing emotional disorders in patients suffering from GAD, ODD, and OCD. The prevalence of general biological vulnerability in patients with emotional disorders was higher than that in normal people. This finding suggests that patients suffering from emotional disorders are biologically and genetically more susceptible to emotional disorders. Additionally, general psychological vulnerability found to be more prevalent in patients with emotional disorders than in normal people. Interestingly, this finding is in line with the premises of triple vulnerability model. Individuals suffering from emotional disorders when encounter threatening challenges and events feel defeated, which indicate their chronic disability in the case of running into negative unpredictable and out of control events. This feeling of not having control over life events is mainly associated with the potential of negative emotional responses. 
Finally, we found a disorder-specific psychological vulnerability is more prevalent in patients suffering from emotional disorders compared with normal people. Having negative, inflexible, ideal, and dysfunctional attitudes toward oneself, others, and the future leads to the development of depression in individuals due to their unrealistic nature. The metacognitive attitude of thought-action fusion leads to the development of dysfunctional attitudes, which might last for hours. As a result, there might be no opportunity for the individual to assess the content of his/her thought and behavior causing the appearance of obsessive-compulsive symptoms. Similarly, the intolerance of uncertainty tends to interpret vague information as threatening, which causes and exacerbates worry and anxiety appearing as GAD in some cases.

\section{Ethical Considerations}

\section{Compliance With Ethical Guideline}

In case of confirmation of diagnosis, after receiving the criteria for inclusion in research and completing the consent form, the research tools completed individually. Normal people were also volunteered from public places in Urmia and after receiving consent.

\section{Funding}

This research did not receive any specific grant from funding agencies in the public, commercial, or not-forprofit sectors.

\section{Conflict of Interest}

The authors declare no conflict of interest.

\section{Acknowledgments}

The authors wish to extend special thanks to the participants of this study who generously shared their precious time. 


\title{
ارزيابى مدل آسيبيذيرى سهكانه در بيشبينى اختلالات هيجانى
}

توحيد رنجبرى'، "جواد كريمى"، ابوالفضل محمدى"، محمدرضا نوروزى"

\author{
ا - دانشجوى كارشئاسى ارشد، تروه روانشناسى، دانشكده روانشناسى و علوم تربيتى، دانشكَاه تهران، تهران، ايران.

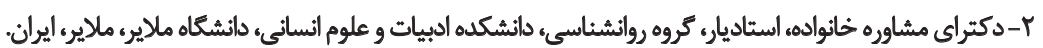

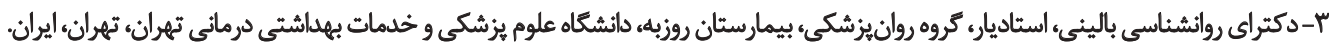

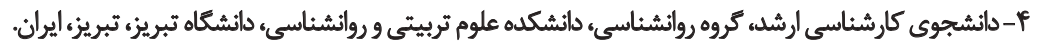

\begin{abstract}
حكטי

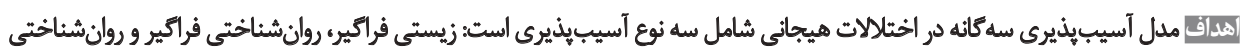

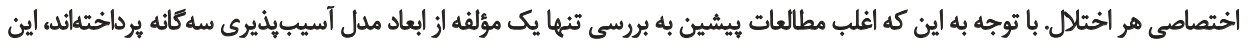

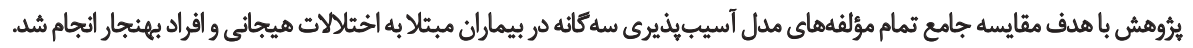

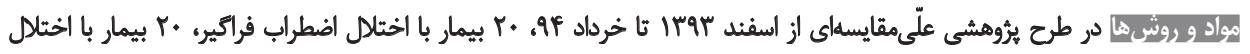

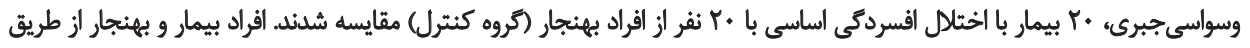

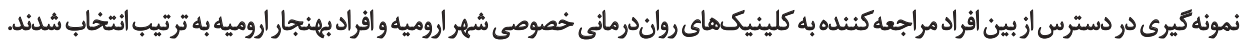

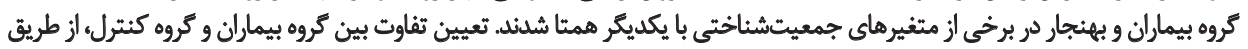

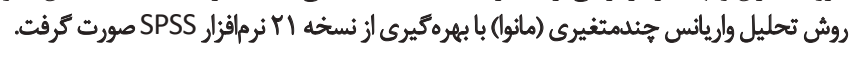

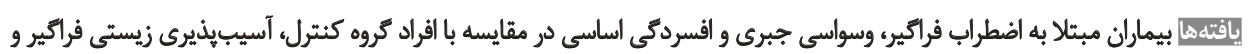

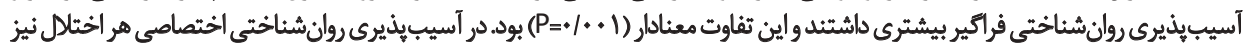

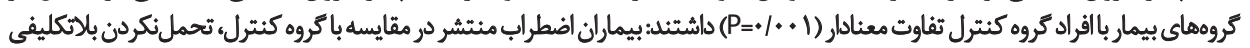

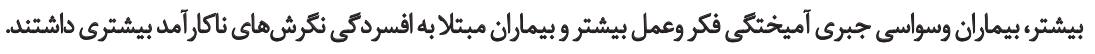

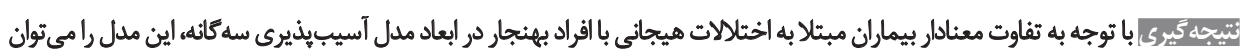
معيار سبب شناختى و تشخيصى جامع و ومناسبى در اين بيمار بان دانست.
\end{abstract}

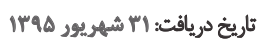

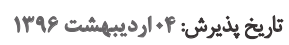

كليدوأوهها:

آسيب يذيرى سه كانه، اخحتلالات هيجانئي، أقراد

بهنجار

اختلال وابسته به مواد و اختلالات افسردگى در رديف جهارم

مقدمه تشخيص هاى روانيزشكى قرار مى كيرد [هـ].

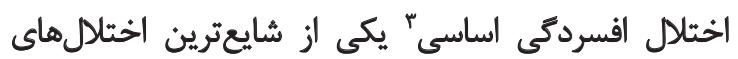

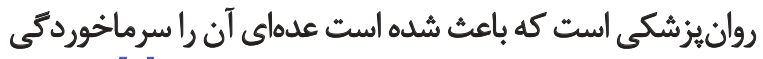

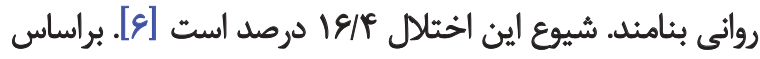

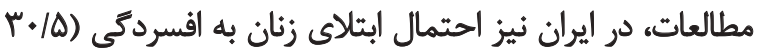

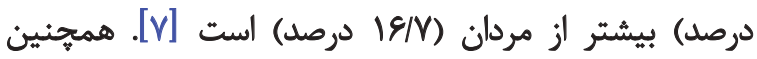

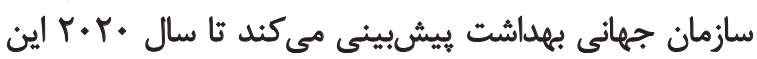

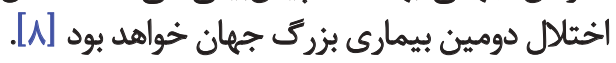

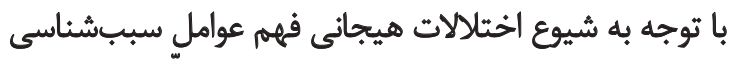
اين اختلالات اهميت بالينى ويرُهاى دارد. عوامل علتّى و زميئلساز

اختلالات اضطرابى از جمله اختلالات هيجانى بسيار شايع

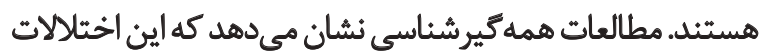

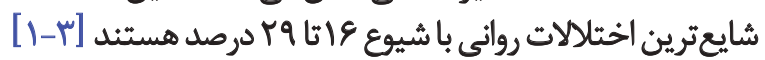

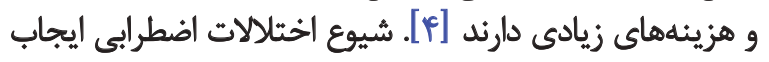

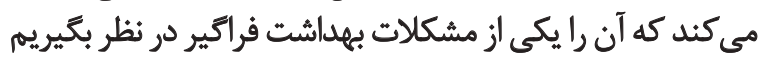

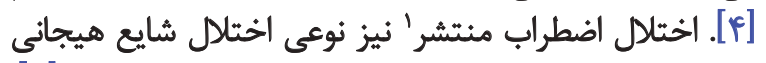

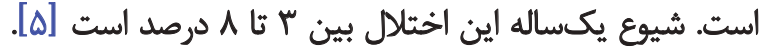

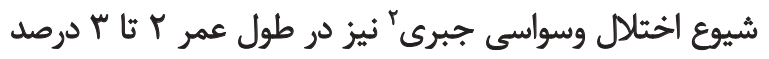

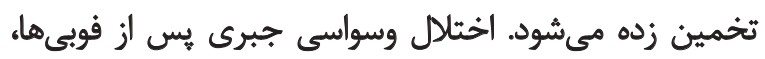

\section{-}

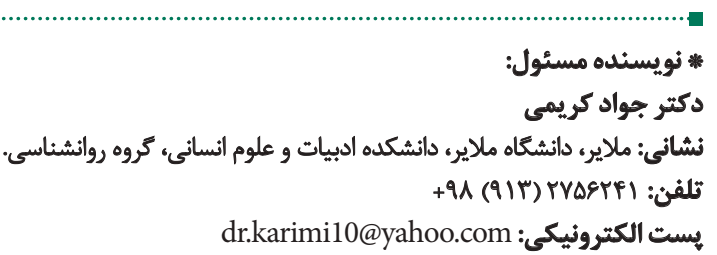




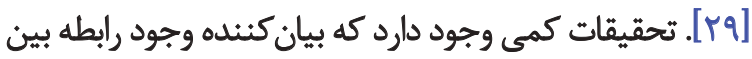

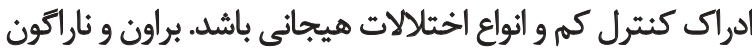

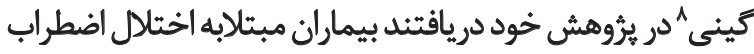

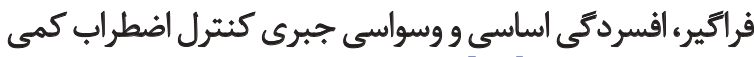

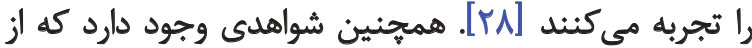

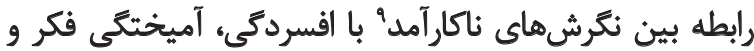

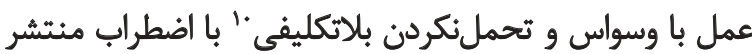

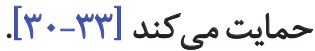

بر اين اساس، مسئله اصلى يُروهش حاضر اين است كه آيا

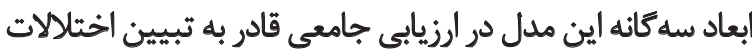

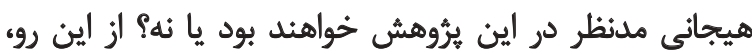

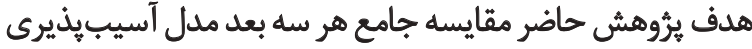

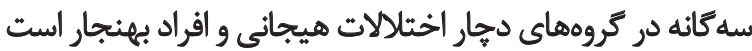

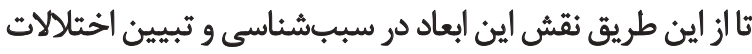

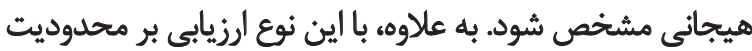

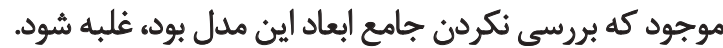

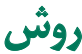

مطالعه حاضر از نوع علّى مقايسهاى و زمان اجراى آن از اسفند

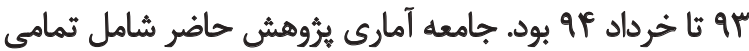

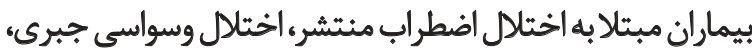

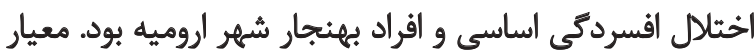

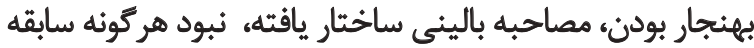

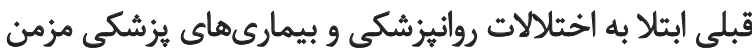

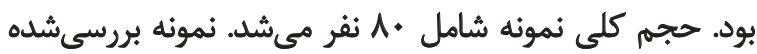

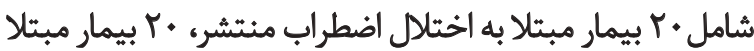

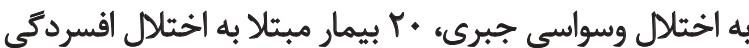

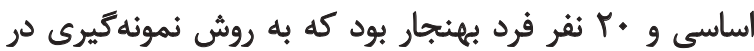

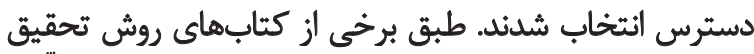

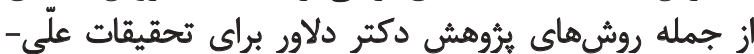

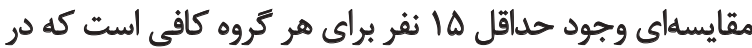

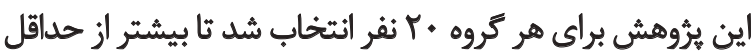

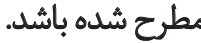

بيماران با توجه به معيارهاي ورود وخروج يُروهش از مراجعان

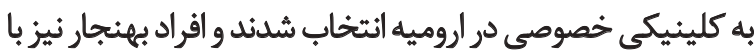

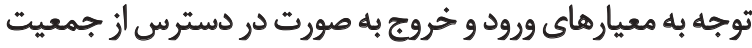

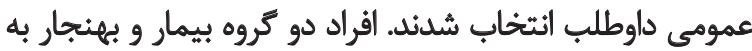

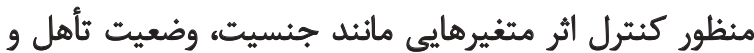

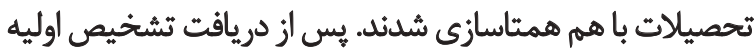

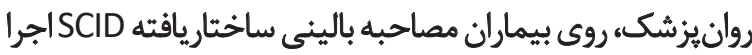

8. Brown \& Naragon-Gainey

9. Dysfunctional attitudes

10. Intolerance of uncertainty
متعددى براى اين اختلالات مطرح شده است؛ از جمله عوامل

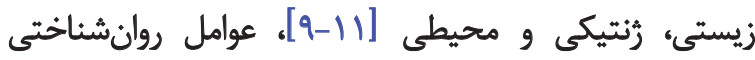

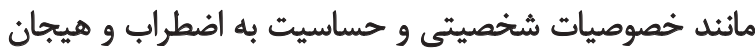

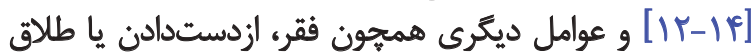

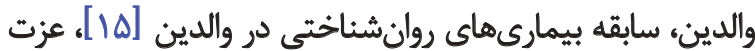

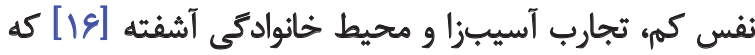

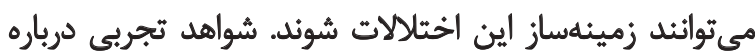

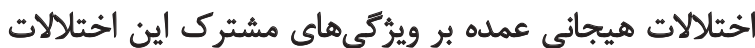

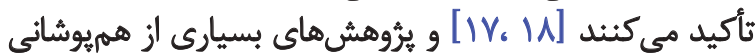

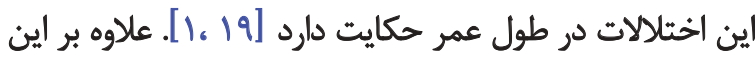

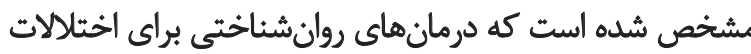

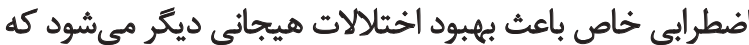

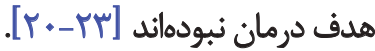

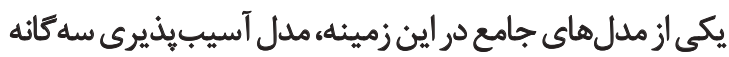

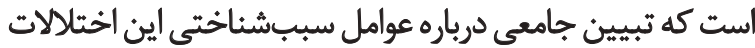

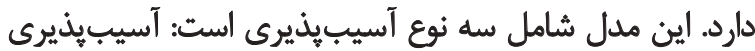

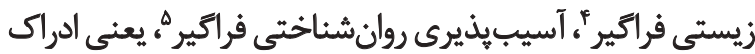

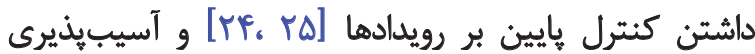

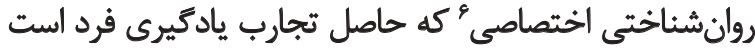

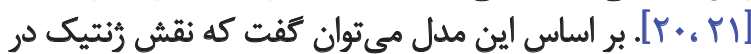

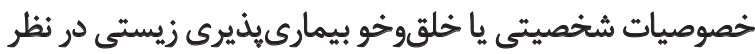

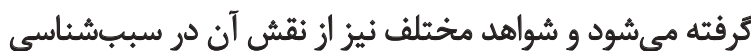

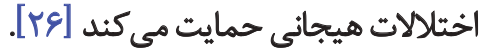

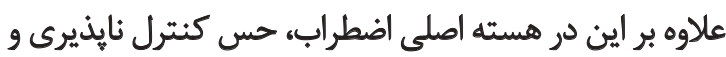

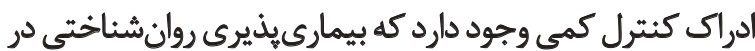

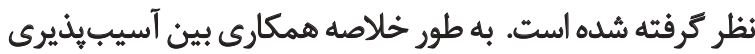

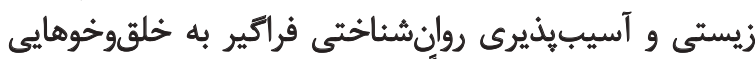

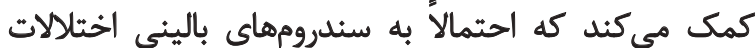

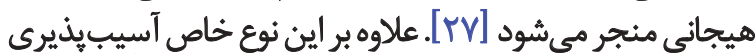

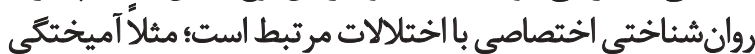

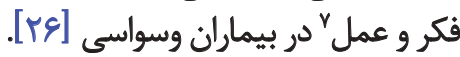

با وجود مشهوربودن اين مدل به عنوان جارجوبى براي درى

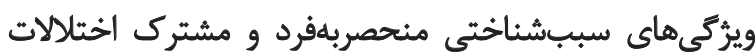

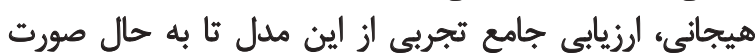

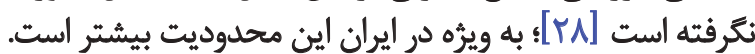

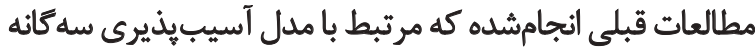

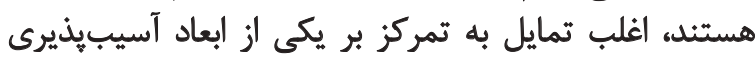

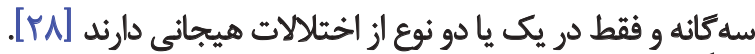

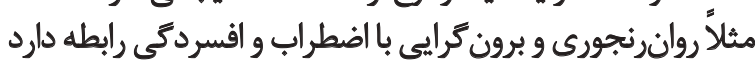

4. General biological vulnerability

5. General psychological vulnerability

6. Disorder-specific psychological vulnerabilities

7. Thought-action fusion 
مقياس برون كرايي و روانرنجورى اين يرسشنامه استفاده شد.

$$
\text { "يرسش نامه كثترل اضطراب" }
$$

در يُروهش حاضر براى اندازمكيرى بعد آسيبيذيرى

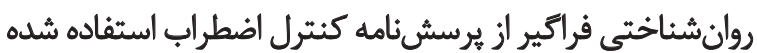

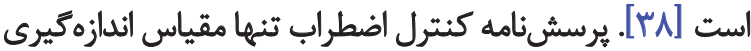

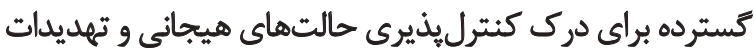

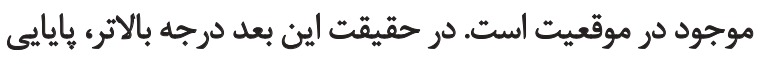

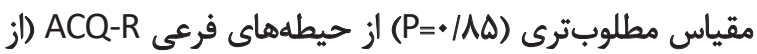

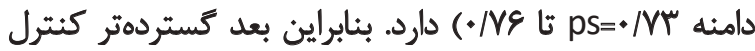

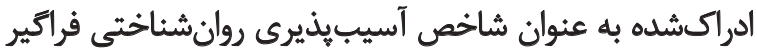

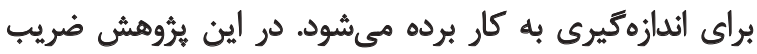

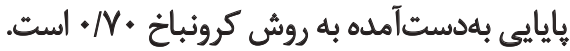

$$
\text { برسش نامه آميخثتكى فكر" }
$$

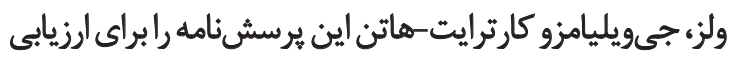

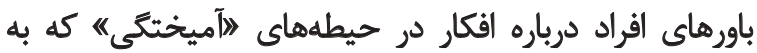

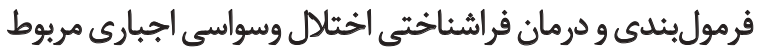

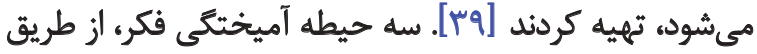

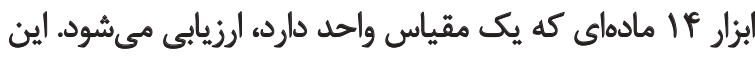

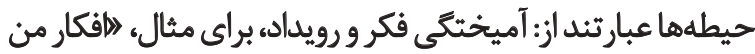

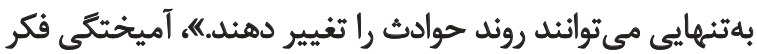

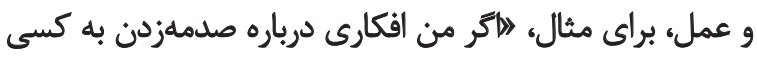

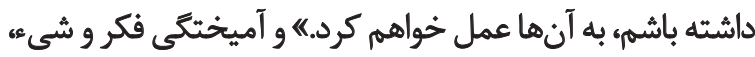
براى مثال، هافكار و خاطرات من مي توائند به اشيا منتقل شونديه

از نظر ساختار عاملى، در مطالعهاى بر روى دانشجويان، بعد إندائ

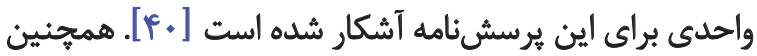

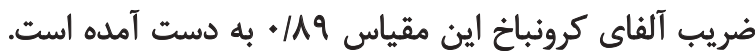

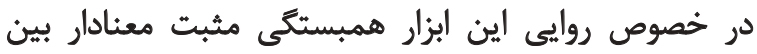

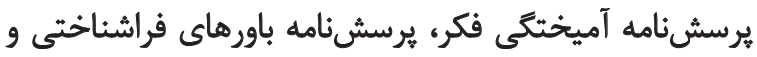

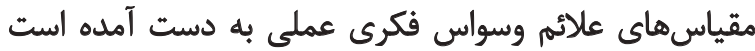

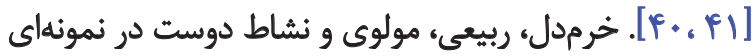

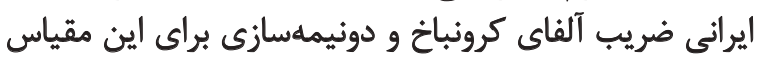

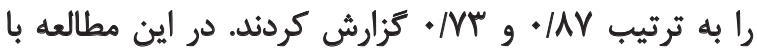

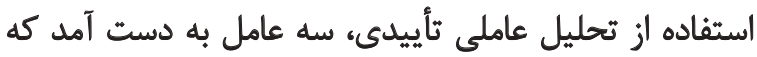

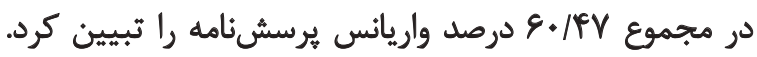

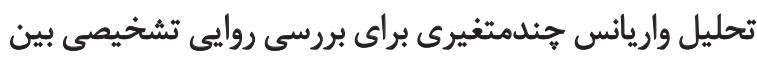

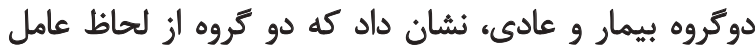

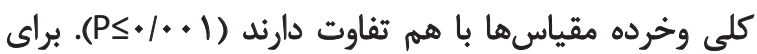

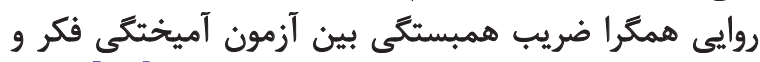

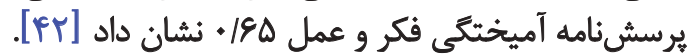

12. Anxiety Control Questionnaire-Revised

13. Thought Fusion Instrument

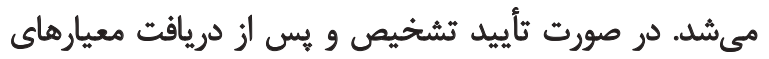

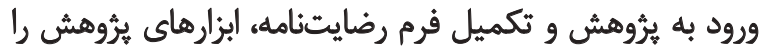

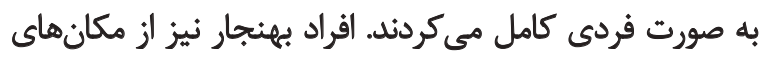

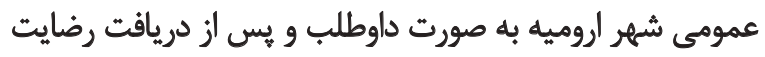

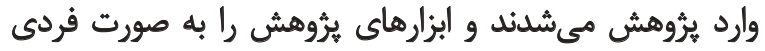

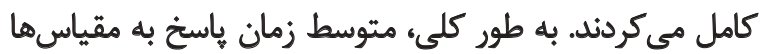

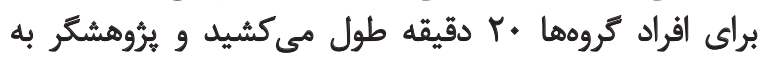

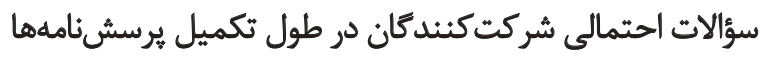

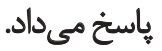

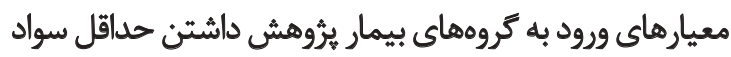

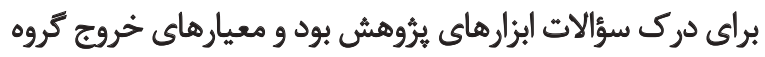

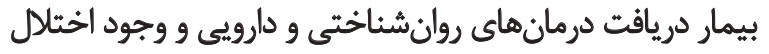

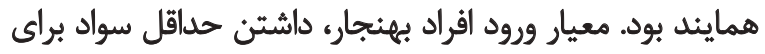

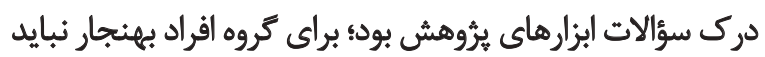

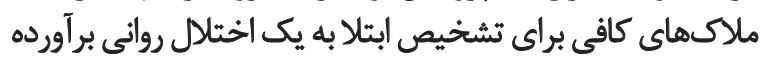

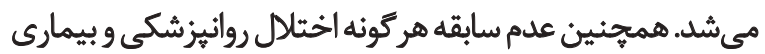

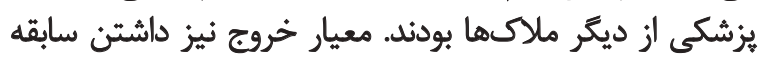

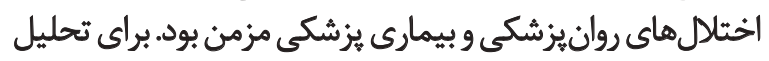

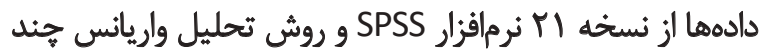

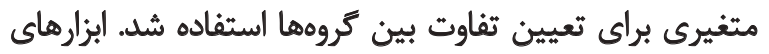
مورد استفاده ازاين قرار بودند:

$$
\text { مقياس ئنج عاملى ئئو }
$$

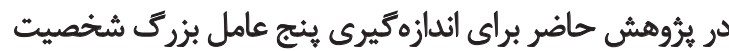

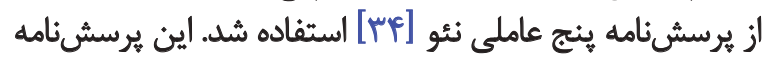

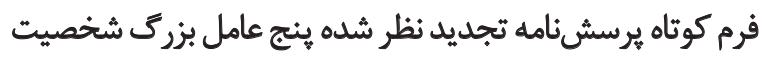

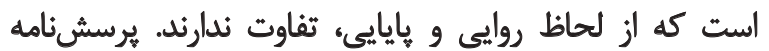

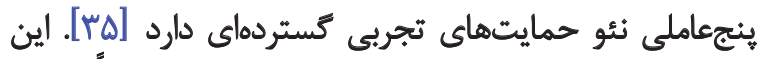

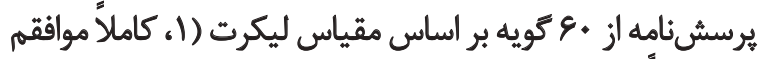

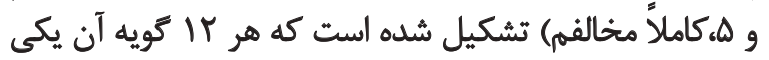

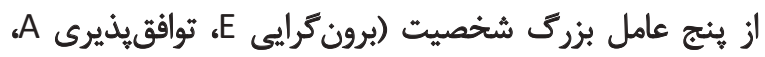

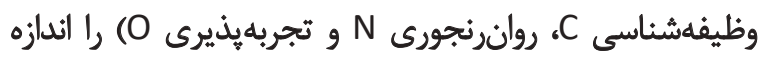

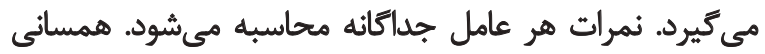

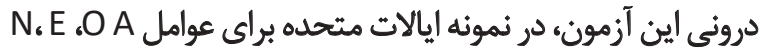

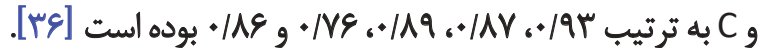

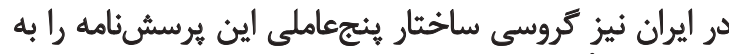

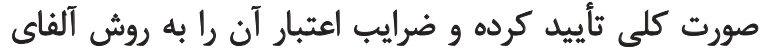

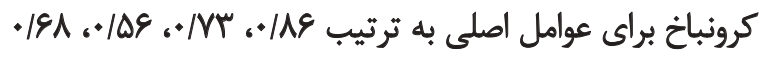

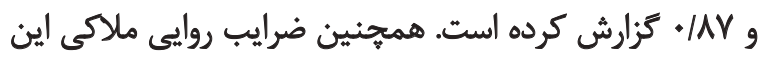

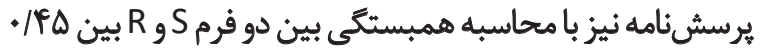

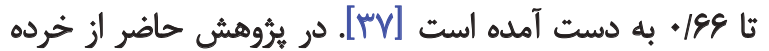

11. NEO-FFI-R 
تعين تفاوت بين كروهها تحليل شدند. قبل از استفاده از

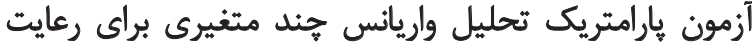

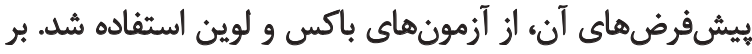

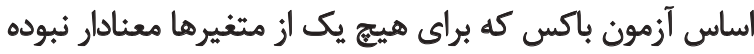

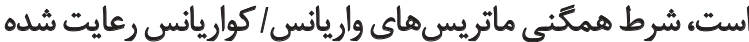

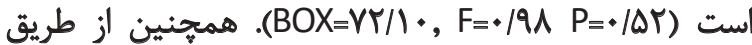

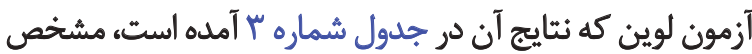

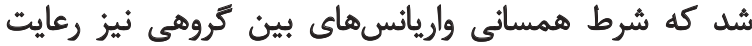

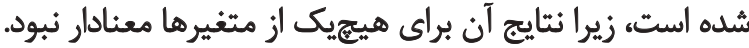

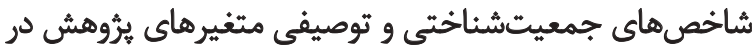
جدول شماره او و ارائه شده است.

جدول شماره f ن نشان ميدهد كه اثر اصلى متغير كروه بر

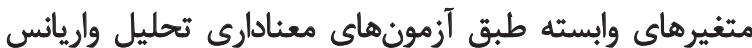

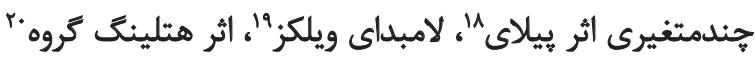

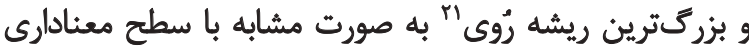

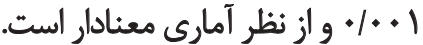

جدول شماره ه نشان مى دهد كه بين افراد جهار كروه تحت

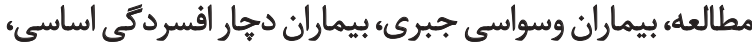

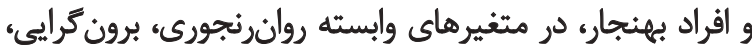

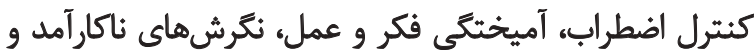

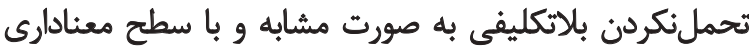

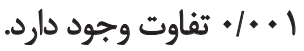

جدول شماره r نشان ميدهد كه ميانگين روانرنجورى اختوان

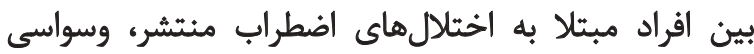

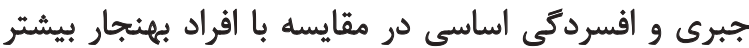

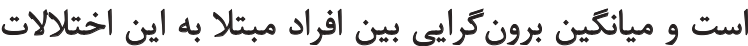

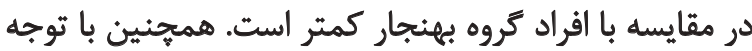

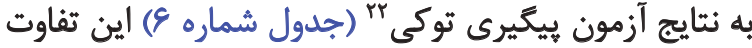

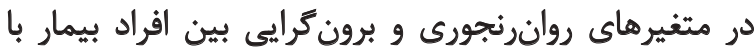

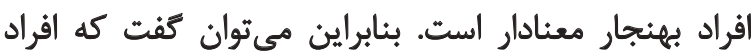

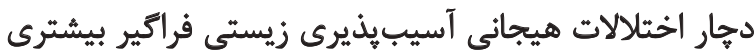

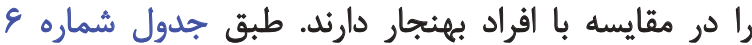

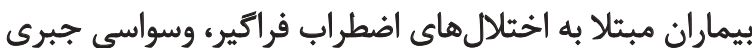

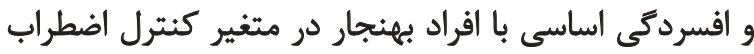

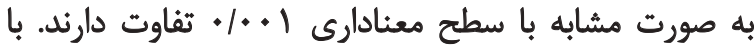

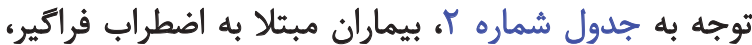

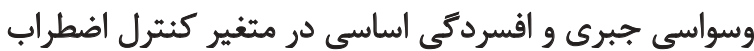

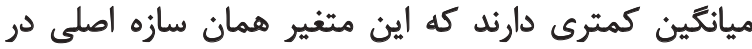

18. Pillai's Trace

19. Wilks' Lambda

20. Hotelling's Trace

21. Roy's Largest Root

22. Tukey's Honest Significant Difference (Tukey's HSD)

$$
\text { مقياس عقايدناكارا آمدئ1 }
$$

از اين مقياس براى سنجش و ارزيابى فرضيات و اعتقادات

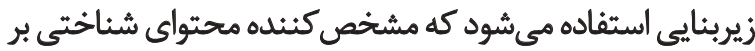

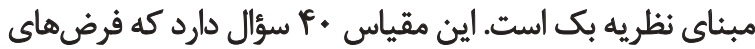

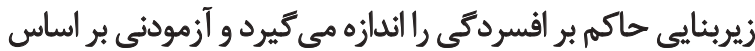

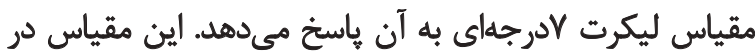

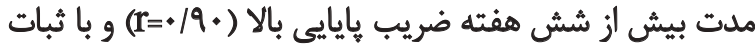

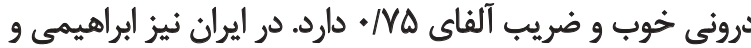

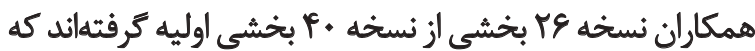

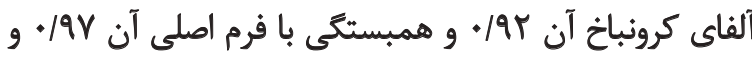

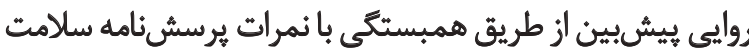

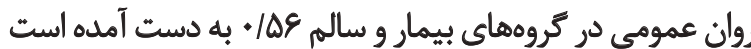

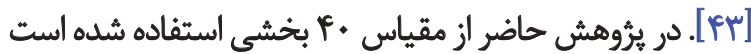

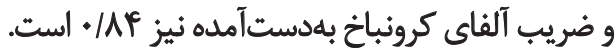

$$
\text { مثياس تحمل نكردن بلاتكليفي } 10
$$

براى جمعآورى اطلاعات مربوط به تحملنكردن بلاتكليفى فئي

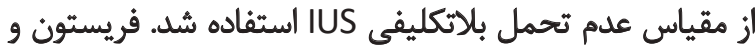

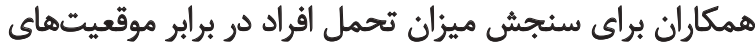

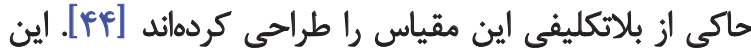

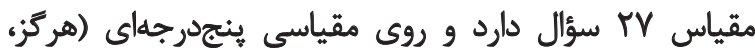

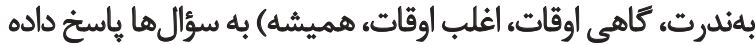
مىشود. نسخه اوليه فرانسوى همساني درونى

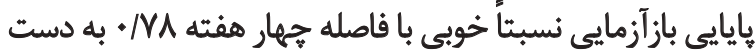
آورده است باست [FF]

باهر و داكاس نسخه انغليسى آن را تهيه و اعتباريابى كردهاند

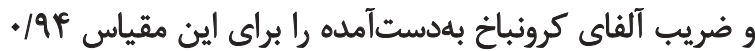

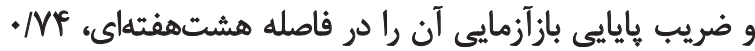

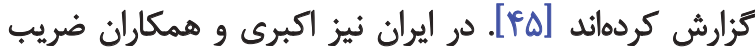

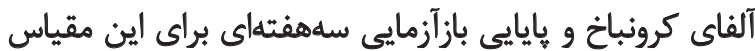

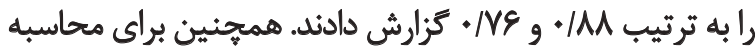

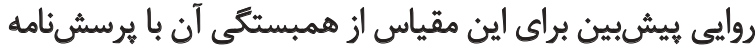

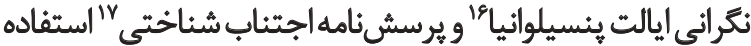

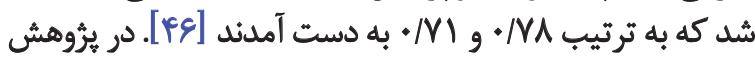

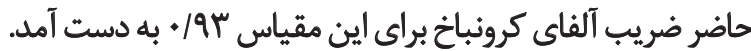

يافتهها

دادهها از طريق روش تحليل واريانس جند متغيرى براى

14. Dysfunctional Beliefs Scale

15. The Intolerance of Uncertainty Scale

16. Penn-State Worry Questionnaire (PSWQ)

17. Cognitive Avoidance Questionnaire (CAQ) 
جدول ا. فراواثى و درصد فراوانى بر اساس جنسيت، وضعيت تأهل و تحصيلات به تفكيك كروه بيمار و بهنجار

\begin{tabular}{|c|c|c|c|c|}
\hline هرصد فراواتى & فراوانى & سطح & مثغير & كروه \\
\hline$r g / N$ & r & هرد & \multirow{2}{*}{ جنسيت } & \multirow{9}{*}{ بيمار } \\
\hline$g \pi / \pi$ & ra & نن & & \\
\hline$\pi / \pi$ & r. & هجرد & \multirow{2}{*}{ وضعيت تأهل } & \\
\hline $\operatorname{sen}$ & p. & مثتاهل & & \\
\hline$r+\%$ & M & زير دييلم & \multirow{5}{*}{ تحصيلات } & \\
\hline reN & $\pi$ & دييلم & & \\
\hline S/V & $r$ & فوق دييلم & & \\
\hline $\mathrm{ra} / \cdot$ & 10 & ل ليسانس & & \\
\hline W & 1 & فوق ليسانس و بالاتر & & \\
\hline$r \omega /$. & $r$ & مرد & \multirow{2}{*}{ جنسيت } & \multirow{9}{*}{ بهنجار } \\
\hline$\varepsilon \Delta /$. & r & زن & & \\
\hline$r .1$ & 8 & 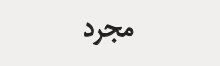 & \multirow{2}{*}{ وضعيت تأهل } & \\
\hline$v . \%$ & 10 & 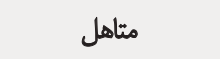 & & \\
\hline$r \%$ & 8 & زير دييلم & \multirow{5}{*}{ تحصيلات } & \\
\hline ro/. & $\checkmark$ & دييلم & & \\
\hline$\Delta / \cdot$ & 1 & فوق دييلم & & \\
\hline $\mathrm{r} \omega /$. & $\Delta$ & ل ليسأس & & \\
\hline$\Delta / \cdot$ & 1 & فوق ليسانس و بالاتر & & \\
\hline
\end{tabular}

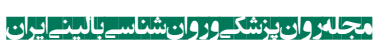

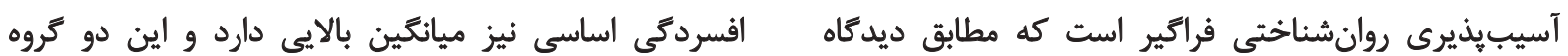

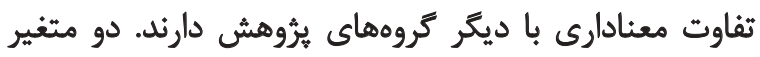

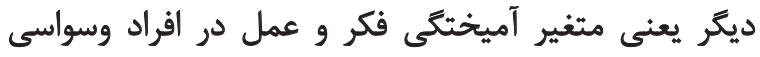

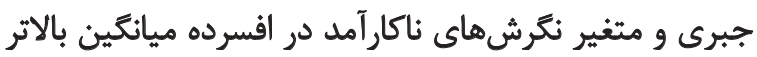

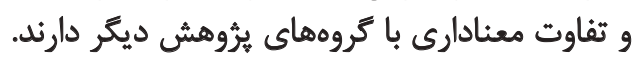

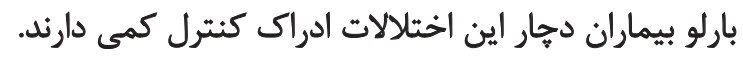

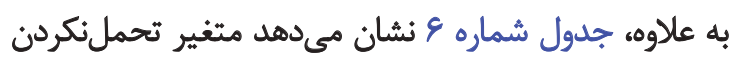

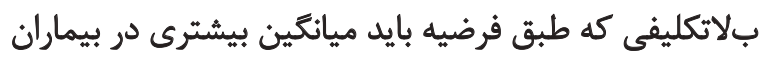

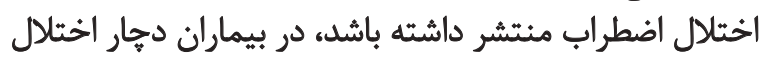

جدول r. ميانكين و انحراف معيار روانرئجورى، برون كرايى، كثترل اضطرابه آميختى فكر و عمل، نترشهاى ناكارآمد و تحملنكردن بلاتكليفى در كروههاى

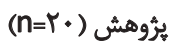

\begin{tabular}{|c|c|c|c|c|c|c|}
\hline عدم تحمل بلاتكليفى & نكرش هاى ناكارآمد & آميختكى فكر وعمل & كتترل اضطراب & برونترايى & رواندرنجورى & \\
\hline (انحراف معيار) & (انحراف معيار) & (انحراف معيار) & (انحراف معيار) & (انحراف معيار) & (انحراف معيار) & \\
\hline$\Delta F / Q \Delta(I F / I T)$ & $119 / 1 f(r+/ 99)$ & $\Lambda \Delta / \Delta \Delta(T \Psi / T T)$ & ra/If(Ir/.9) & $r / Q \Delta(F / N G)$ & $F \cdot / f \cdot(\varepsilon / Y q)$ & $O C D$ \\
\hline 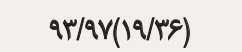 & $\mid \varepsilon+/ F \Delta(T F / M I)$ & $F \Delta / F+(19 / 1 \Delta)$ & $r \& / \Delta \cdot(1+/ \Delta+)$ & $F \cdot /$ NA(NIY) & $P V / \& V\left(V / T^{2} q\right)$ & MDD \\
\hline$A V / \cdot \Delta(\mid V / V \Lambda)$ & $I T r / N q\left(Y \Delta / M^{q}\right)$ & $T r / \& \Delta(T r / Q \Lambda)$ & $\mu E / R T(1) / \cdot 1)$ & $r+18 \Delta(\Delta / \Delta q)$ & $r+/ L Q(\Delta / q T)$ & GAD \\
\hline$\Delta \Delta / A Y(I S / \mu F)$ & $/ \Delta \Delta(I V / A Y)$ & $M \varphi / \Psi \otimes(I S / M T)$ & $g F / \Lambda \cdot(1) / M)$ & $P V / \cdot \cdot(r / A V)$ & $\mu+/ 1 \cdot(\Delta / \Lambda+)$ & بهنجار \\
\hline
\end{tabular}


جدول ب. نتايج آزمون لوين درباره بيشفرض همسائي واريانس هاي جهار كروه در مثغيرهاي يروهش

\begin{tabular}{|c|c|c|c|c|}
\hline سطح معنادارى & dff & df $)$ & $F$ & متغير \\
\hline $.18 V$ & ve & $r$ & .101 & روانرنجورى \\
\hline.$/ I V$ & ve & $r$ & $1 / M^{\infty}$ & برون كرايى \\
\hline 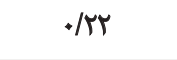 & ve & $r$ & $1 / p q$ & كثترل اضطراب \\
\hline 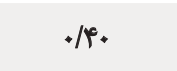 & ve & $r$ &.$/ 4$ & آميختكى فكر - عمل \\
\hline oft & ve & $r$ &.$/ 2 \Delta$ & نكرش هاي ناكارآمد \\
\hline.$/ 18$ & ve & $r$ & $I / V \Delta$ & عدم تحمل بلاتكليفي \\
\hline
\end{tabular}

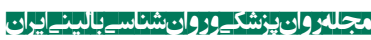

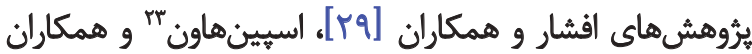

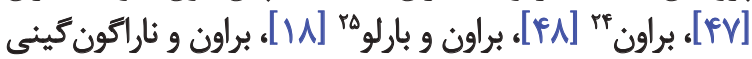

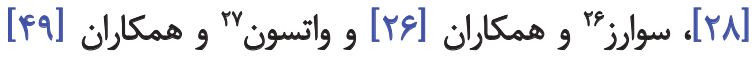

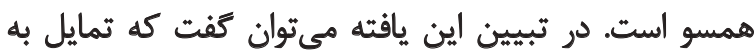

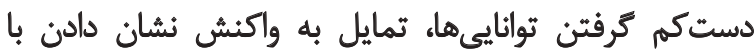

23. Spinhoven

24. Brown

25. Brown \& Barlow

26. Suárez

27. Watson

بنا بر اطلاعات بهدستآمده از اين يُؤوهش، مى دوان كفت كه

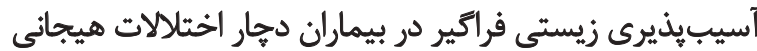

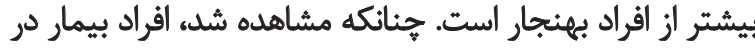

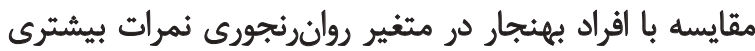

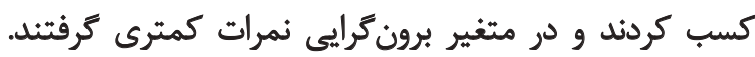

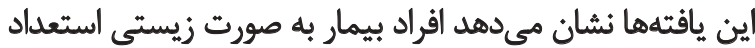

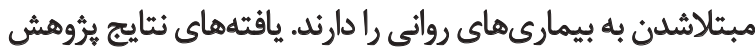

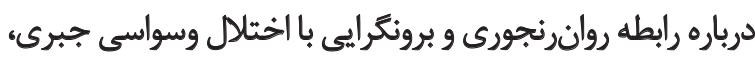
اختلال افسردگى اساسى و اختلال اضطراب منتشر با نتايج

جدول F. نتايج آزمونهاي معثادارى تحليل واريانس جئد متغيرى (MANOVA) براى اثر اصلى متغير كروه بر متغيرهاى وابسته

\begin{tabular}{|c|c|c|c|c|}
\hline سطح معنادارى & $F$ & الرزئ & نوع آزمون & متغير \\
\hline $.1+.1$ & $q / p$ & VRr & اثر ييلايع & \multirow{4}{*}{ كروه } \\
\hline $1+.1$ & $\mathrm{~W} / \cdot \mathrm{A}$ &.$M$ & لاهبياي ويلكز & \\
\hline$+\cdots 1$ & $I r / I V$ & $r / M$ & اثر هتلينى كروه & \\
\hline$\% 1$ & $r+180$ & r & بزركترين ريشه روى & \\
\hline
\end{tabular}

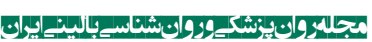

جدول هـ نتايج تحليل واريانس جثندمثيري آثار كروه بر متغيرهاي وابسته

\begin{tabular}{|c|c|c|c|c|c|}
\hline سطح معنادارى & $F$ & ميانكين مجذورات & $d f$ & مجموع مجذورات & متغير وإبسته \\
\hline .10 .1 & rE/Aq & I.FT/AT & $r$ & MITAW & روانونجنورى \\
\hline $.1 . .1$ & $\mathrm{IV/M}$ & $811 / 9$. & $r$ & MFA/V. & برون كرايى \\
\hline $.1 . .1$ & $r+19 u$ & MrAP/EF & $r$ & Rar/ar & كثترل اضطر|ب \\
\hline $.1+.1$ & MT/MP & IfTEV//AF & $r$ & PMrAfIFT & آميختكي فكر و عمل \\
\hline $.1+.1$ & IfIF & nmrar & $r$ & $r r+. r / a r$ & نكَرش هاي ناكارأمد \\
\hline$+1 *+1$ & $r E / T+$ & $n+4+|A|$ & $r$ & Trrar/fy & تحمل نكردن بلاتكليفى \\
\hline
\end{tabular}


جدول و نتايج آزمون بينيكيرى توكى

\begin{tabular}{|c|c|c|c|c|c|}
\hline سطح معنادارى & خطاى اسثاندارد & تفاوت مياتكين (1-T) & r Fروه r & كروها & مثغير \\
\hline$\%+r$ & $1 / q$ & $-V / T V$ & MDD & & \multirow{6}{*}{ روانرنجورى } \\
\hline.$/ 99$ & $1 / 98$ &.$/ \pi r$ & GAD & $O C D$ & \\
\hline $.1 . .1$ & $1 / 9$ & $1 . \pi$ & افراد بهنجار & & \\
\hline$\mu r$ & $1 / 9$ & V/ar & GAD & MDD & \\
\hline $.1 . .1$ & $1 / Q$ & IV/AY & افراد بهنجار & & \\
\hline $.1+.1$ & $1 / q$ & 1.1 .0 & افراد بهنجار & GAD & \\
\hline$\cdot / \pi r$ & I/AP & $r / \Delta \Delta$ & MDD & & \multirow{6}{*}{ برون كرايى } \\
\hline .119 & I/AF & $1 / r$ & GAD & $O C D$ & \\
\hline $.1 .+1$ & $I / A F$ & $-q / D$ & افراد بهنجار & & \\
\hline.$|8|$ & I/Ar & $-T / T A$ & GAD & MDD & \\
\hline$\%+1$ & $V / A F$ & $-\mid Y / 9$. & افراد بهنجار & & \\
\hline $.1 . .1$ & I/AF & $-1 . / \% \Delta$ & افراد بهنجار & GAD & \\
\hline.$/ 9$ & $r / q \Delta$ & $T / 98$ & MDD & & \multirow{6}{*}{ كتترل اضطراب } \\
\hline . $/ \Delta T^{\prime}$ & $r / q \Delta$ & $-\Delta / \pi f$ & GAD & $O C D$ & \\
\hline $.1+.1$ & r/qه & -rQ//Q & افراد بهنجار & & \\
\hline.$/ 19$ & $r / q \Delta$ & $-V / 99$ & GAD & MDD & \\
\hline $.1 . .1$ & $r / q \Delta$ & $-Y N T^{-}$ & افراد بهنجار & & \\
\hline.$/ .+1$ & $r / q \Delta$ & $-r \cdot / \mu \cdot$ & افراد بهنجار & GAD & \\
\hline $.1 .+1$ & s/99 & rVqם & MDD & & \multirow{6}{*}{ ميختكى فكر- عمل } \\
\hline $.1 . .1$ & $8 / 99$ & $\Delta T / R$. & GAD & $O C D$ & \\
\hline.. .1 & $8 / 99$ & $s \cdot / v$ & افراد بهنجار & & \\
\hline 每 & 9199 & $I T / N \Delta$ & GAD & MDD & \\
\hline .1 .1 & 9199 & $r V / \infty$ & افراد بهنجار & & \\
\hline .19. & 8/99 & Nr. & افراد بهنجار & GAD & \\
\hline $.1 .+1$ & $v / \cdot v$ & $-(r) / \%$. & MDD & & \multirow{6}{*}{ نكرش هاي ناكارآمد } \\
\hline.$/ \mathrm{V}$ & $v / \cdot v$ & $-14 / \% \Delta$ & GAD & $O C D$ & \\
\hline.$/ 99$ & $v / \cdot v$ & $-1 / 4$ & افراد بهنجار & & \\
\hline /..r & $v / \cdot v$ & rEIFA & GAD & MDD & \\
\hline $.1++1$ & $V / \cdot V$ & rqq. & افراد بهنجار & & \\
\hline.$/ M F$ & $V / \cdot v$ & Ir/Tf & افراد بهنجار & GAD & \\
\hline
\end{tabular}




\begin{tabular}{|c|c|c|c|c|c|}
\hline سطح معنادارى & خطاى استاندارد & تغاوت ميانكين (1-r) & r r & كروه 1 & متغير \\
\hline $.1 . .1$ & $\Delta / \pi$ & $-r q / . r$ & MDD & & \multirow{6}{*}{ عدم تحمل بالاتكليفى } \\
\hline $.1 . .1$ & $\Delta / \pi r$ & $-r g /$. & GAD & $O C D$ & \\
\hline.$/ 99$ & $\Delta / \pi r$ &.$- / 9 r$ & اقراد بهنجار & & \\
\hline 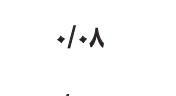 & $\Delta / \pi r$ & IT/ar & GAD & MDD & \\
\hline $.1 .+1$ & $\Delta / \pi T$ & rNl. & افراد بهنجار & & \\
\hline $.1+.1$ & $\Delta / \pi T$ & $r \Delta / M$ & افراد بهنجار & GAD & \\
\hline
\end{tabular}

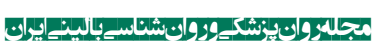

اشاره كرد. عامل آزادكننده كورتيكوترويين محور هييوتالاموس

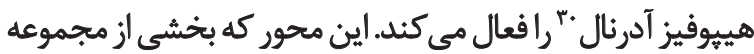

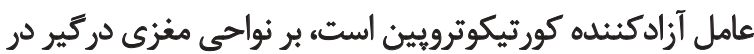

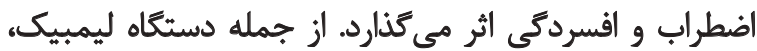

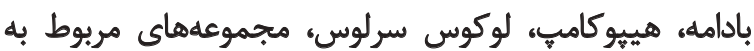

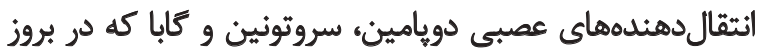

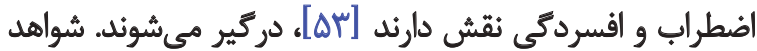
يُؤهشى نيز از اين ديدكاه كه فعاليت محور

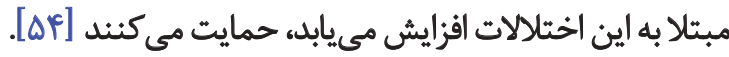
يافته ديكر بروهش نشان داد كه آسيبيذيرى روانشناختى آناخي

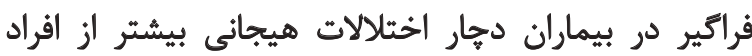

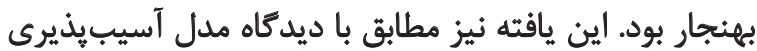

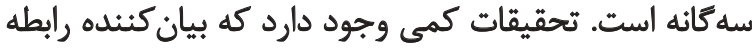

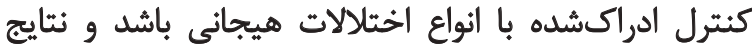

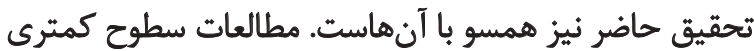

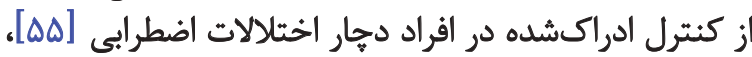

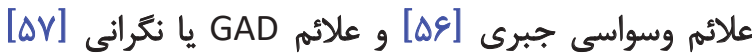

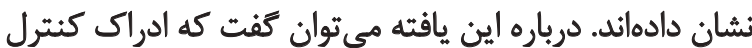

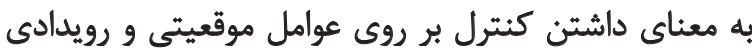

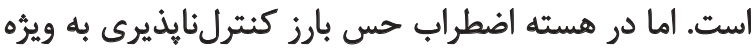

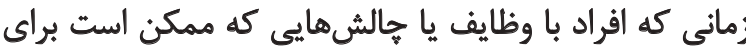

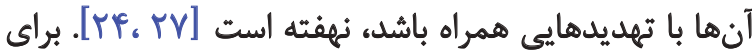

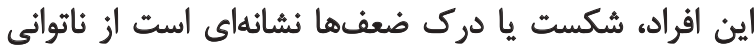

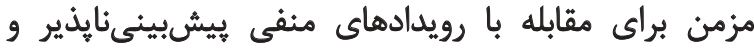

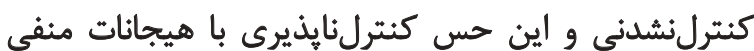

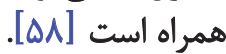
از طرف ديگر افراد سالم ادراك كنترل بيشترى دارند و در

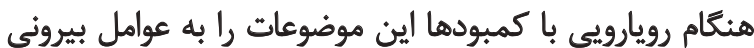

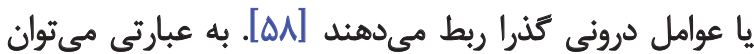

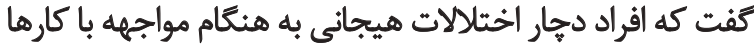

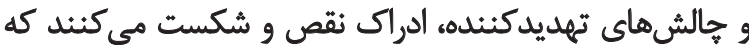

30. Hypothalamic-Pituitary-Adrenal (HPA)
هيجانات شديد و خود انتقادى در موقعيتهاى فشارزا از جمله

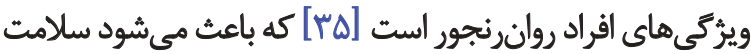
روان كمتر و مشكلات روانشناختى بيشترى داشتئه باشند.

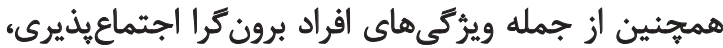

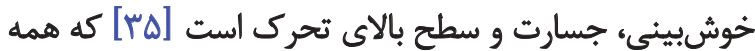

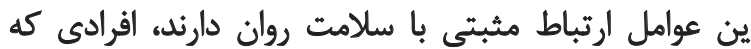

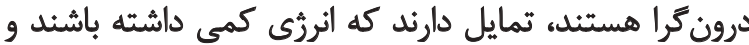

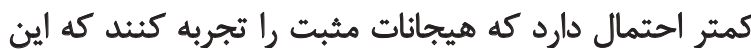

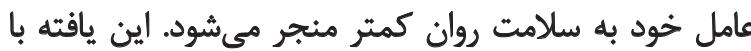

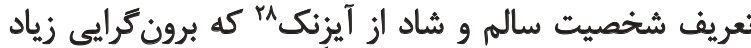

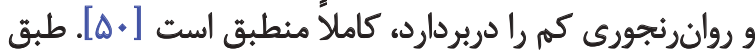

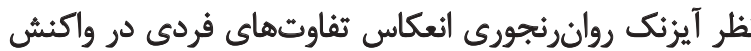

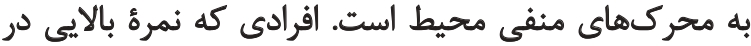

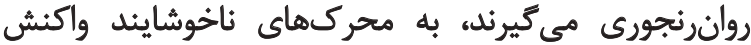

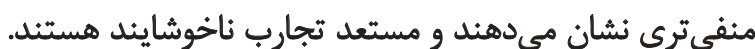

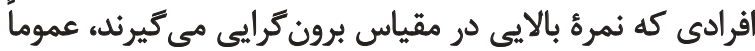

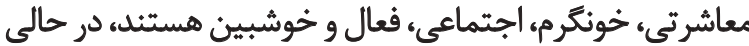

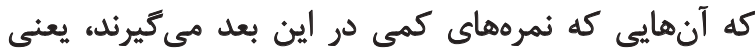

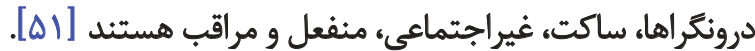
همجئين درباره اين يافته مى توان بيان كرد كه بُروهشهاي

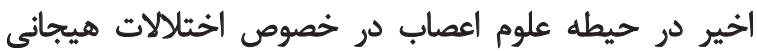

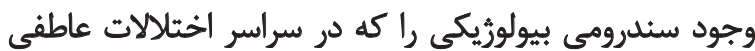

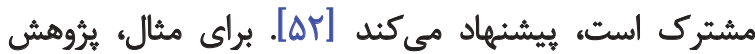

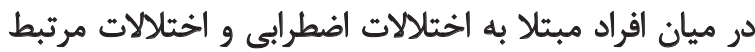

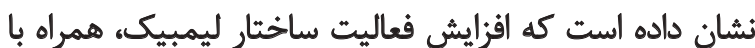

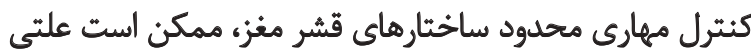

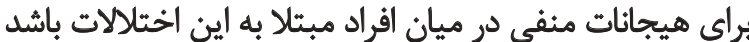

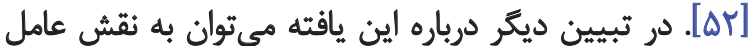

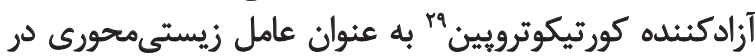

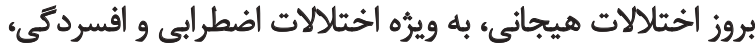

29. Corticotropin-Releasing Factor (CRF) 


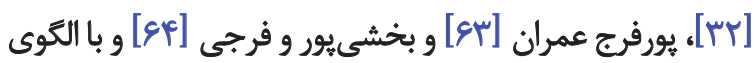

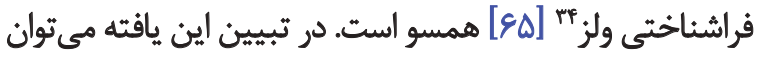

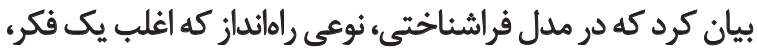

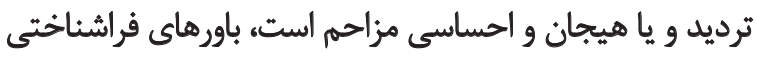

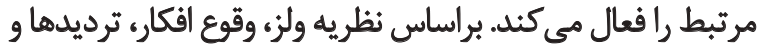

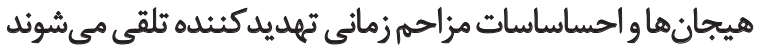

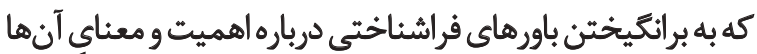

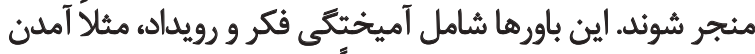

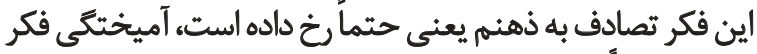

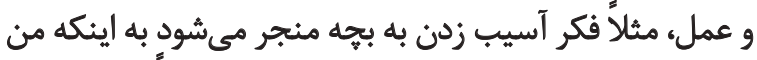

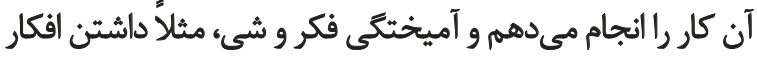

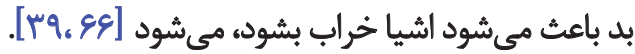

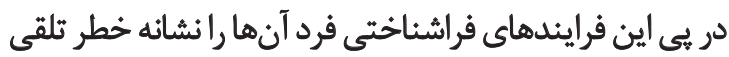

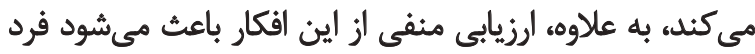

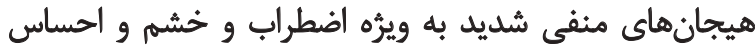

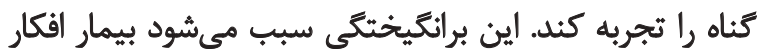

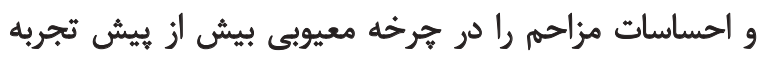

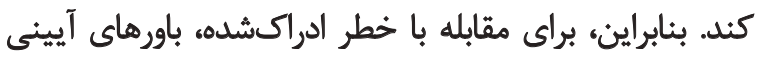

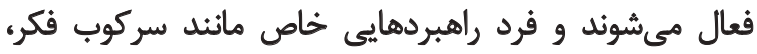

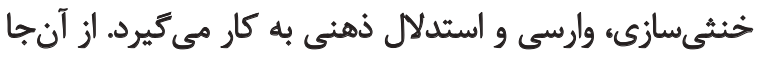

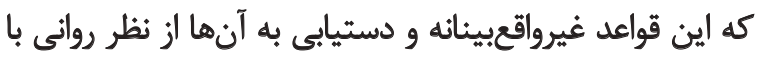

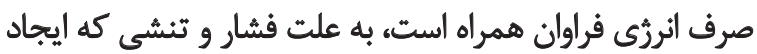

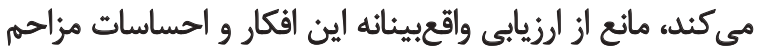

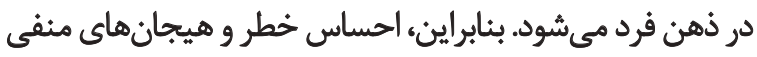

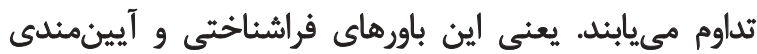

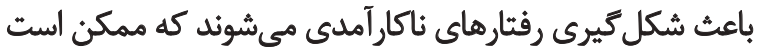

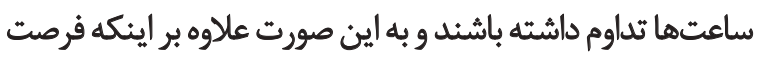

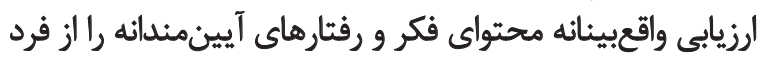

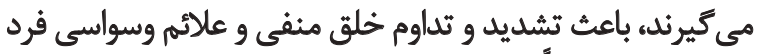

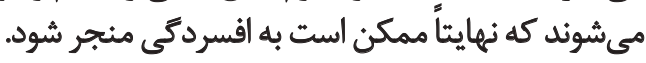

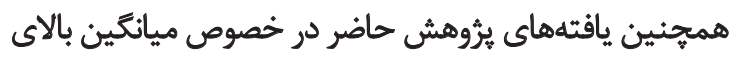

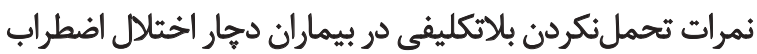

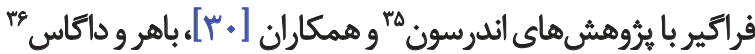

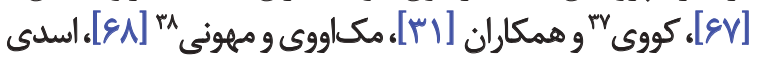

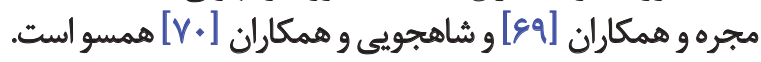

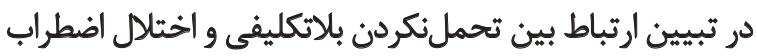

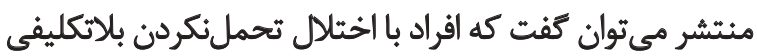

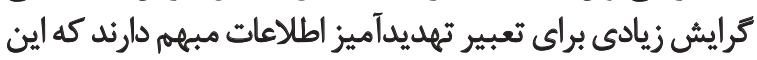

34. Wells

35. Anderson

36. Buhr \& Dugas

37. Cowie

38. McEvoy \& Mahoney
بيانكر ناتوانى مزمن آنها در هنتام مقابله با رويدادهاي منفى كني

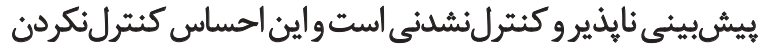

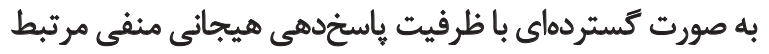

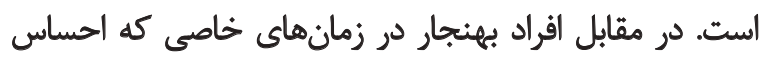

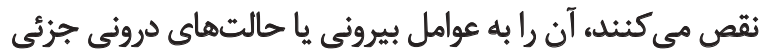

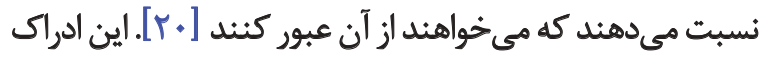

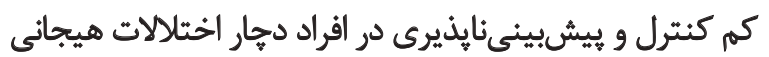

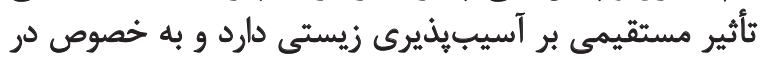

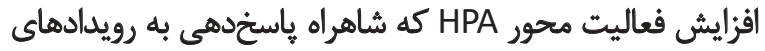

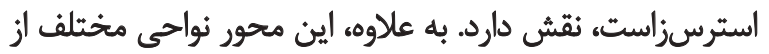

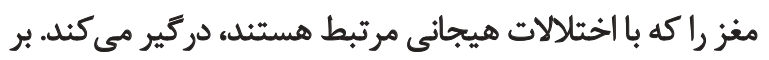

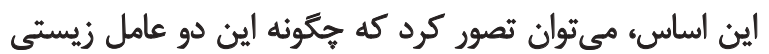

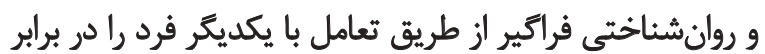

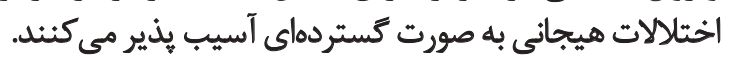

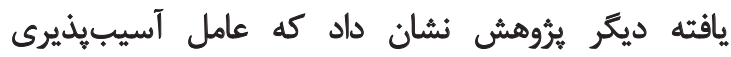

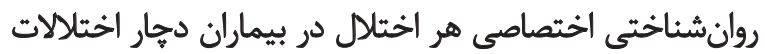

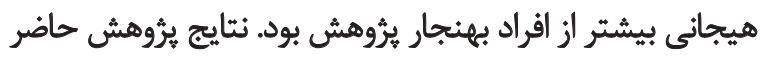

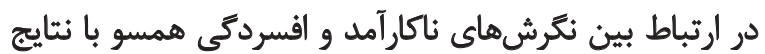

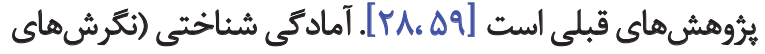

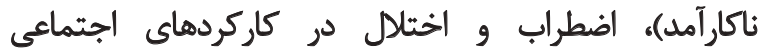

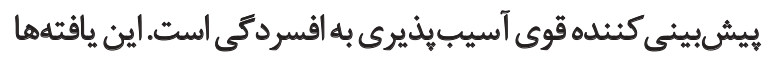

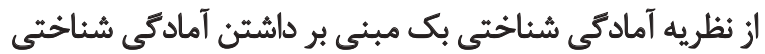

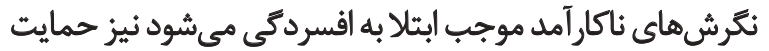

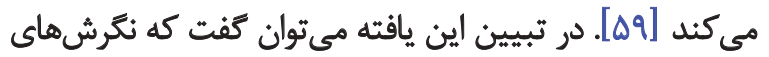

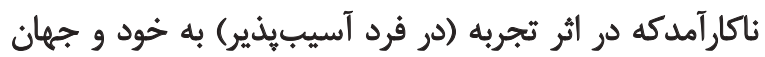

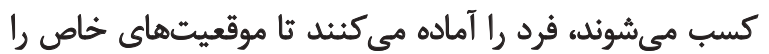

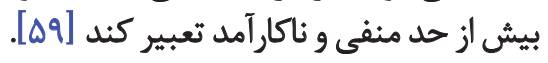

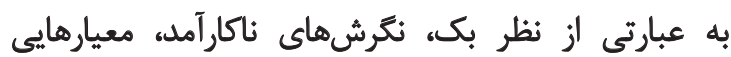

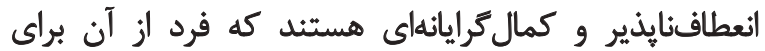

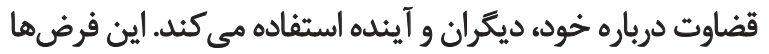

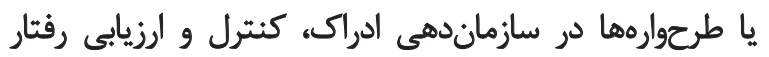

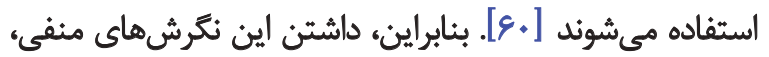

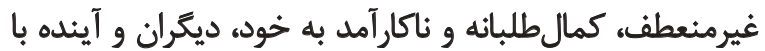

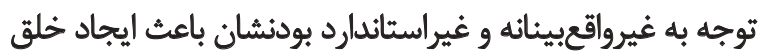

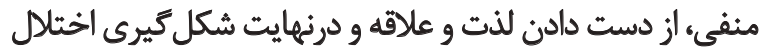
افسردگى در اين افراد مىشوند.

يافتههاى ثُروهش حاضر، درباره ميانكين بالاتر آميختئى

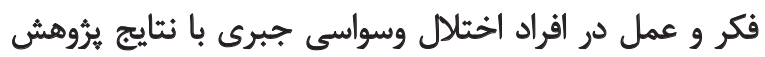

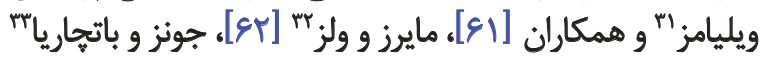

31. Williams

32. Myers \& Wells

33. Jones \& Bhattacharya 
ممكن است باعث افزايش نكرانى و اضطراب درباره تعبير مفاهيم

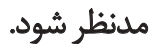

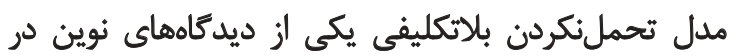

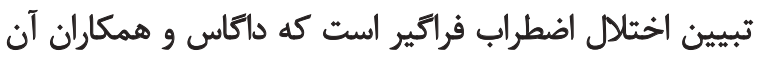

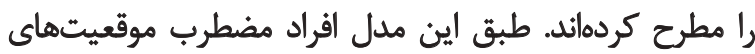

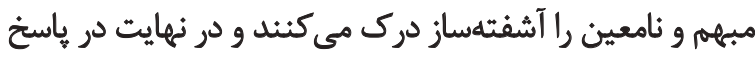

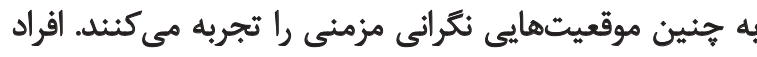

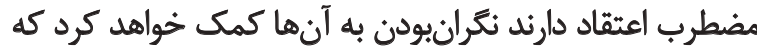

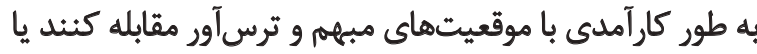

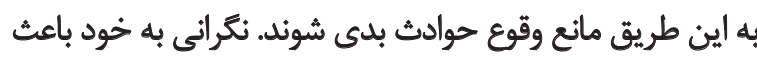

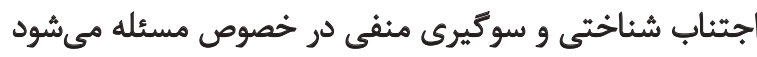

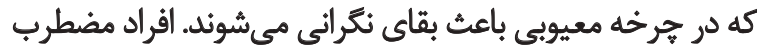

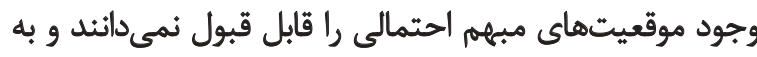

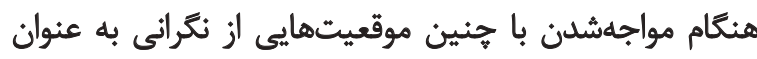

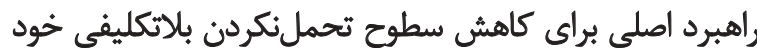

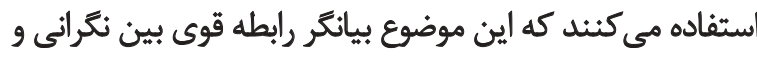
تحمل تكردن بلاتكليفي است.

\section{تتيجهيَ}

دستاوردهاى يُوهش حاضر رامي توان ماني معيارى به منظور سبب

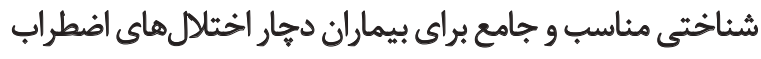

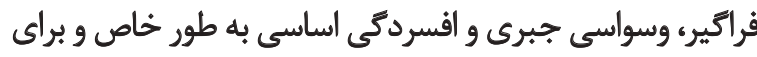

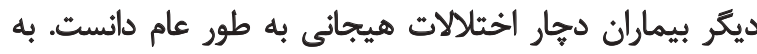

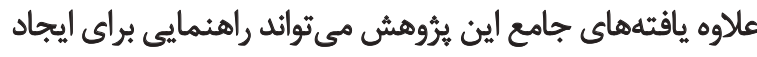

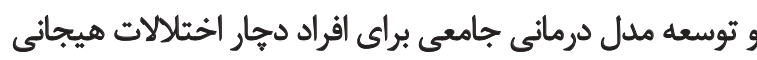

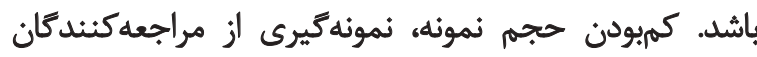

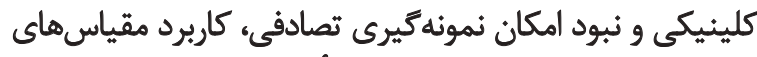

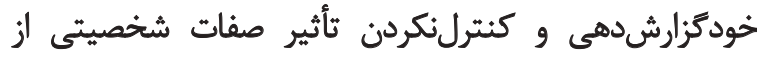

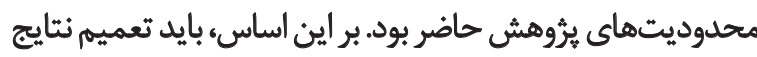

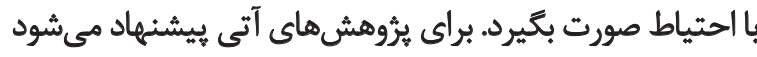

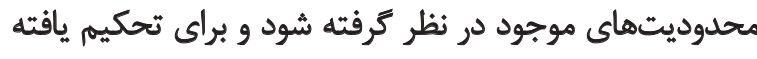

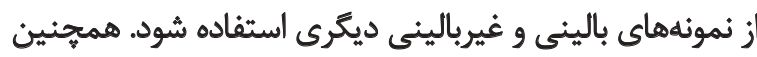

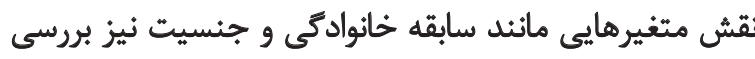

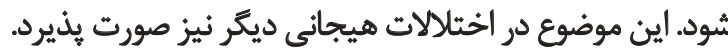

$$
\text { سباسكَّز إلى }
$$

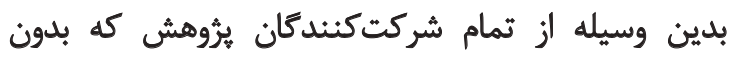

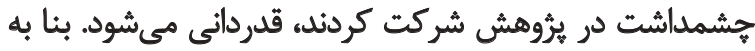

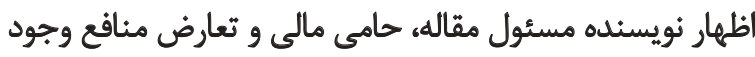




\section{References}

[1] Kessler RC, Berglund P, Demler O, Jin R, Merikangas KR, Walters EE. Lifetime prevalence and age-of-onset distributions of DSM-IV disorders in the National Comorbidity Survey Replication. Archives of General Psychiatry. 2005; 62(6):593-602. doi: 10.1001/archpsyc.62.6.593

[2] Kessler RC, Chiu WT, Demler O, Merikangas KR, Walters EE. Prevalence, severity, and comorbidity of 12-month DSM-IV disorders in the National Comorbidity Survey Replication. Archives of General Psychiatry. 2005; 62(6):617-27. doi: 10.1001/ archpsyc.62.6.617

[3] Kessler RC, Aguilar-Gaxiola S, Alonso J, Chatterji S, Lee S, Ormel J, et al. The global burden of mental disorders: An update from the WHO World Mental Health (WMH) surveys. Epidemiologia e Psichiatria Sociale. 2009; 18(1):23-33. doi: 10.1017/ s1121189x00001421

[4] Costa e Silva JA. The public health impact of anxiety disorders: a WHO perspective. Acta Psychiatrica Scandinavica. 1998; 98(s393):2-5. doi: 10.1111/j.1600-0447.1998.tb05959.x

[5] Sadock BJ, Sadock VA. Kaplan and Sadock's synopsis of psychiatry: Behavioral sciences/clinical psychiatry. Philadelphia, Pennsylvania: Lippincott Williams \& Wilkins; 2007.

[6] Kessler RC, Gruber M, Hettema JM, Hwang I, Sampson N, Yonkers KA. Co-morbid major depression and generalized anxiety disorders in the National Comorbidity Survey followup. Psychological Medicine. 2008; 38(3):365-74. doi: 10.1017/ s0033291707002012

[7] Mirzadehahari Z, Mohammadi F, Eini zinab H, Khosravi M, mousavi N, Agasi M. [Survey of association between major depression disorder in women and household food insecurity $(\mathrm{Pr}-$ sian)]. Iranian Journal of Nutrition Sciences \& Food Technology. 2015; 10(1):9-20.

[8] Kenny MA, Williams JMG. Treatment-resistant depressed patients show a good response to Mindfulness-based Cognitive Therapy. Behaviour Research and Therapy. 2007; 45(3):617-25. doi: 10.1016/j.brat.2006.04.008

[9] Hettema JM, Prescott CA, Myers JM, Neale MC, Kendler KS. The structure of genetic and environmental risk factors for anxiety disorders in men and women. Archives of General Psychiatry. 2005; 62(2):182-9. doi: 10.1001/archpsyc.62.2.182

[10] Karayiorgou M, Altemus M, Galke BL, Goldman D, Murphy $\mathrm{DL}$, Ott J, et al. Genotype determining low catechol-O-methyltransferase activity as a risk factor for obsessive-compulsive disorder. Proceedings of the National Academy of Sciences. 1997; 94(9):4572-5. doi: 10.1073/pnas.94.9.4572

[11] Vink D, Aartsen MJ, Schoevers RA. Risk factors for anxiety and depression in the elderly: A review. Journal of affective disorders. 2008;106(1):29-44. doi: 10.1016/j.jad.2007.06.005

[12] American Psychiatric Association. Diagnostic and statistical manual of mental disorders (DSM-5®). Arlington: American Psychiatric Association Publishing; 2013.

[13] Clark LA, Watson D, Mineka S. Temperament, personality, and the mood and anxiety disorders. Journal of Abnormal Psychology. 1994; 103(1):103. doi: 10.1037//0021-843x.103.1.103
[14] Leahy RL, Holland SJ, McGinn LK. Treatment plans and interventions for depression and anxiety disorders. New York: Guilford Press; 2011.

[15] Zhang X, Norton J, Carriere I, Ritchie K, Chaudieu I, Ancelin $\mathrm{M}$. Risk factors for late-onset generalized anxiety disorder: results from a 12-year prospective cohort (The ESPRIT study). Translational Psychiatry. 2015; 5(3):e536. doi: 10.1038/tp.2015.31

[16] Blanco C, Rubio J, Wall M, Wang S, Jiu CJ, Kendler KS. Risk factors for anxiety disorders: Common and specific effects in a national sample. Depression and Anxiety. 2014; 31(9):756-64. doi: $10.1002 /$ da. 22247

[17] Brown TA, Barlow DH. Classification of anxiety and mood disorders. In: Barlow DH, editor. Anxiety and Its Disorders: The Nature and Treatment of Anxiety and Panic. New York: Guilford Press; 2002.

[18] Brown TA, Barlow DH. A proposal for a dimensional classification system based on the shared features of the DSM-IV anxiety and mood disorders: Implications for assessment and treatment. Psychological Assessment. 2009; 21(3):256-71. doi: 10.1037/ a0016608

[19] Brown TA, Campbell LA, Lehman CL, Grisham JR, Mancill RB. Current and lifetime comorbidity of the DSM-IV anxiety and mood disorders in a large clinical sample. Journal of Abnormal Psychology. 2001;110(4):585-99. doi: 10.1037//0021843x.110.4.585

[20] Allen L, White K, Barlow D, Shear MK, Gorman J, Woods S. Cognitive-Behavior Therapy (CBT) for panic disorder: Relationship of anxiety and depression comorbidity with treatment outcome. Journal of Psychopathology and Behavioral Assessment. 2010; 32(2):185-92. doi: 10.1007/s10862-009-9151-3

[21] Borkovec T, Abel JL, Newman H. Effects of psychotherapy on comorbid conditions in generalized anxiety disorder. Journal of Consulting and Clinical Psychology. 1995; 63(3):479-83. doi $10.1037 / / 0022-006 x .63 .3 .479$

[22] Tsao JCI, Lewin MR, Craske MG. The effects of cognitive-behavior therapy for panic disorder on comorbid conditions. Journal of Anxiety Disorders. 1998; 12(4):357-71. doi: 10.1016/s08876185(98)00020-6

[23] Tsao JCI, Mystkowski JL, Zucker BG, Craske MG. Effects of cognitive-behavioral therapy for panic disorder on comorbid conditions: Replication and extension. Behavior Therapy. 2002 33(4):493-509. doi: 10.1016/s0005-7894(02)80013-2

[24] Barlow DH. Unraveling the mysteries of anxiety and its disorders from the perspective of emotion theory. American Psychologist. 2000; 55(11):1247-63. doi: 10.1037//0003-066x.55.11.1247

[25] Barlow DH. Disorders of emotion. Psychological Inquiry. 1991; 2(1):58-71. doi: 10.1207/s15327965pli0201_15

[26] Suárez L, Bennett S, Goldstein C, Barlow DH. Understanding anxiety disorders from a "triple vulnerability" framework. In Antony MM, Stein MB, editors. Oxford Handbook of Anxiety and Related Disorders. Oxford: Oxford University Press; 2009.

[27] Barlow DH. Anxiety and its disorders: The nature and treatment of anxiety and panic. New York: Guilford Press; 2002.

[28] Brown TA, Naragon-Gainey K. Evaluation of the unique and specific contributions of dimensions of the triple vulnerability model to the prediction of DSM-IV anxiety and mood disorder 
constructs. Behavior Therapy. 2013; 44(2):277-92. doi: 10.1016/j. beth.2012.11.002

[29] Afshar H, Roohafza H, Hassanzadeh-Keshteli A, Sharbafchi MR, Feizi A, Adibi P. Association of personality traits with psychological factors of depression, anxiety, and psychological distress: a community based study. International Journal of Body, Mind and Culture. 2015; 2(2):105-14.

[30] Anderson KG, Dugas MJ, Koerner N, Radomsky AS, Savard $\mathrm{P}$, Turcotte J. Interpretive style and intolerance of uncertainty in individuals with anxiety disorders: A focus on generalized anxiety disorder. Journal of Anxiety Disorders. 2012; 26(8):823-32. doi: 10.1016/j.janxdis.2012.08.003

[31] Cowie J, Clementi MA, Alfano CA. Examination of the Intolerance of Uncertainty Construct in Youth With Generalized Anxiety Disorder. Journal of Clinical Child \& Adolescent Psychology. 2016:1-9. doi: 10.1080/15374416.2016.1212358

[32] Jones R, Bhattacharya J. A role for the precuneus in thoughtaction fusion: Evidence from participants with significant obsessive-compulsive symptoms. NeuroImage: Clinical. 2014; 4:11221. doi: $10.1016 /$ j.nicl.2013.11.008

[33] Thomas J, Altareb B. Cognitive vulnerability to depression: an exploration of dysfunctional attitudes and ruminative response styles in the United Arab Emirates. Psychology and Psychotherapy: Theory, Research and Practice. 2012; 85(1):117-21. doi: 10.1111/j.2044-8341.2011.02015.x

[34] Costa PT, McCrae RR. Normal personality assessment in clinical practice: The NEO Personality Inventory. Psychological Assessment. 1992; 4(1):5-13. doi: 10.1037/ /1040-3590.4.1.5

[35] McCrae RR, Costa Jr PT. The five-factor theory of personality. In: John OP, Robins RW, Pervin LA, editors. Handbook of Personality: Theory and Research. New York: Guilford Press; 2008.

[36] Costa PT, McCrae RR. Four ways five factors are basic. Personality and Individual Differences. 1992; 13(6):653-65. doi: 10.1016/0191-8869(92)90236-i

[37] Garoosi MT. [New approaches to personality assessment: Application of factor analysis in personality studies (Persian)]. Tabriz: Jamepazhooh; 2001.

[38] Brown TA, White KS, Forsyth JP, Barlow DH. The structure of perceived emotional control: Psychometric properties of a revised anxiety control questionnaire. Behavior Therapy. 2004; 35(1):7599. doi: 10.1016/s0005-7894(04)80005-4

[39] Wells A. Metacognitive therapy for anxiety and depression. New York: Guilford Press; 2009.

[40] Gwilliam P, Wells A, Cartwright-Hatton S. Dose meta-cognition or responsibility predict obsessive-compulsive symptoms: a test of the metacognitive model. Clinical Psychology \& Psychotherapy. 2004; 11(2):137-44. doi: 10.1002/cpp.402

[41] Myers SG, Wells A. Obsessive-compulsive symptoms: the contribution of metacognitions and responsibility. Journal of Anxiety Disorders. 2005; 19(7):806-17. doi: 10.1016/j.janxdis.2004.09.004

[42] Khoramdel K, Rabiee M, Molavi H, Neshat-Doost H. [A study psychometric properties of Thought Fusion Instrument (TFI) in student (Persian)]. Iranian Journal of Psychiatry and Clinical Psychology. 2010; 16(1):74-8

[43] Ebrahimi A, Neshatdoost H, Kalantari M, Molavi H, Asadollahi G. [Contributions of dysfunctional attitude scale and gen- eral health subscales to prediction and odds ratio of depression (Persian)]. Journal of Shahrekord University of Medical Sciences 2003; 9(4):8-52.

[44] Freeston MH, Rhéaume J, Letarte H, Dugas MJ, Ladouceur R. Why do people worry? Personality and Individual Differences. 1994; 17(6):791-802. doi: 10.1016/0191-8869(94)90048-5

[45] Buhr K, Dugas MJ. The intolerance of uncertainty scale: psychometric properties of the English version. Behaviour Research and Therapy. 2002; 40(8):931-45. doi: 10.1016/s0005-7967(01)00092-4

[46] Akbari M HH, Andoz Z. [Predictors of worry in student (Persian)]. Paper presented at: The $5^{\text {th }}$ National Seminar on Student's Mental Health. 12-13 May 2010; Tehran, Iran.

[47] Spinhoven P, Elzinga BM, van Hemert AM, de Rooij M, Penninx BW. A longitudinal study of facets of extraversion in depression and social anxiety. Personality and Individual Differences. 2014; 71:39-44. doi: 10.1016/j.paid.2014.07.014

[48] Brown TA. Temporal course and structural relationships among dimensions of temperament and DSM-IV anxiety and mood disorder constructs. Journal of Abnormal Psychology. 2007; 116(2):313-28. doi: 10.1037/0021-843x.116.2.313

[49] Watson D, Gamez W, Simms LJ. Basic dimensions of temperament and their relation to anxiety and depression: A symptombased perspective. Journal of Research in Personality. 2005; 39(1):46-66. doi: 10.1016/j.jrp.2004.09.006

[50] Grossarth-Maticek R, Eysenck HJ. Personality, stress and disease: Description and validation of a new inventory. Psychological Reports. 1990; 66(2):355-73. doi: 10.2466/pr0.1990.66.2.355

[51] Matthews G, Gilliland K. The personality theories of HJ Eysenck and JA Gray: A comparative review. Personality and Individual Differences. 1999; 26(4):583-626. doi: 10.1016/s01918869(98)00158-5

[52] Barlow DH, Sauer-Zavala S, Carl JR, Bullis JR, Ellard KK. The nature, diagnosis, and treatment of neuroticism. Clinical Psychological Science. 2013; 2(3):344-65. doi: 10.1177/2167702613505532

[53] Barlow D, Durand V. Abnormal psychology: an integrative ap proach. Boston, Massachusetts: Cengange Publisher; 2014.

[54] Fischer S, Cleare A. HPA axis functioning as a predictor of psychotherapy response in patients with depression and anxiety disorders-A systematic review and meta-analysis. Psychoneuroendocrinology. 2015; 61:23-4. doi: 10.1016/j.psyneuen.2015.07.454

[55] Gallagher MW, Bentley KH, Barlow DH. Perceived contro and vulnerability to anxiety disorders: A meta-analytic review. Cognitive Therapy and Research. 2014; 38(6):571-84. doi: 10.1007/ s10608-014-9624-x

[56] Moulding R, Kyrios M, Doron G, Nedelikovic M. Mediated and direct effects of general control beliefs on obsessive compulsive symptoms. Canadian Journal of Behavioural Science/Revue canadienne des sciences du comportement. 2009; 41(2):84-92. doi: 10.1037/a0014840

[57] Chapman LK, Kertz SJ, Woodruff-Borden J. A structural equation model analysis of perceived control and psychological distress on worry among African American and European American young adults. Journal of Anxiety Disorders. 2009; 23(1):69-76. doi: 10.1016/j.janxdis.2008.03.018

[58] Paulus DJ, Vanwoerden S, Norton PJ, Sharp C. From neuroticism to anxiety: Examining unique contributions of three transdi- 
agnostic vulnerability factors. Personality and Individual Differences. 2016; 94:38-43. doi: 10.1016/j.paid.2016.01.012

[59] Kërqeli A, Kelpi M, Tsigilis N. Dysfunctional attitudes and their effect on depression. Procedia - Social and Behavioral Sciences. 2013; 84:196-204. doi: 10.1016/j.sbspro.2013.06.534

[60] Jarrett RB, Minhajuddin A, Borman PD, Dunlap L, Segal ZV, Kidner CL, et al. Cognitive reactivity, dysfunctional attitudes, and depressive relapse and recurrence in cognitive therapy responders. Behaviour Research and Therapy. 2012; 50(5):280-6. doi: 10.1016/j.brat.2012.01.008

[61] Williams AD, Lau G, Grisham JR. Thought-action fusion as a mediator of religiosity and obsessive-compulsive symptoms. Journal of Behavior Therapy and Experimental Psychiatry. 2013; 44(2):207-12. doi: 10.1016/j.jbtep.2012.09.004

[62] Myers SG, Wells A. An experimental manipulation of metacognition: A test of the metacognitive model of obsessive-compulsive symptoms. Behaviour Research and Therapy. 2013; 51(4):177-84. doi: 10.1016/j.brat.2013.01.007

[63] Pourfaraj Omran M. [Comparison of Thought-Action Fusion (TAF) in obsessive -compulsive disorder (OCD), depression and comorbidity of this two disorders and normal group (Persian)]. Quarterly Journal of Psychological Studies. 2009; 5(1):35-50.

[64] Bakhshipour Roodsari A, Faraji R. [Relationship between thought-action fusion with obsessive-compulsive symptoms in patients with obsessive-compulsive disorder (Persian)]. Contemporary Psychology. 2011; 5(2):15-22.

[65] Wells A. Emotional disorders and metacognition: Innovative cognitive therapy. Hoboken, New Jersey: John Wiley \& Sons; 2000 .

[66] Wells A. Cognitive therapy of anxiety disorders: A practice manual and conceptual guide. Hoboken, New Jersey: John Wiley \& Sons; 1997.

[67] Buhr K, Dugas MJ. Fear of emotions, experiential avoidance, and intolerance of uncertainty in worry and generalized anxiety disorder. International Journal of Cognitive Therapy. 2012; 5(1):117. doi: 10.1521/ijct.2012.5.1.1

[68] McEvoy PM, Mahoney AE. To be sure, to be sure: Intolerance of uncertainty mediates symptoms of various anxiety disorders and depression. Behavior Therapy. 2012; 43(3):533-45. doi: 10.1016/j. beth.2011.02.007

[69] Asadi S, Abedini M, Poursharifi H, Nikokar M. [The relationship between intolerance of uncertainty and rumination with worry on student population (Persian)]. Journal of Clinical Psychology. 2013; 4(4):83-92.

[70] Shahjoee T, Mahmood Aliloo M, Bakhshipour Roodsari A, Fakhari A. [Intolerance of uncertainty and worry among patients with generalized anxiety disorder and obsessive-compulsive disorder (Persian)]. Iranian Journal of Psychiatry and Clinical Psychology. 2012; 17(4):304-12. 